\title{
HOMOLOGY OF BRAID GROUPS AND THEIR GENERALIZATIONS
}

\author{
VLADIMIR V. VERSHININ \\ Sobolev Institute of Mathematics, Novosibirsk, 630090, Russia \\ E-mail:versh@math.nsc.ru
}

\begin{abstract}
In the paper we give a survey of (co)homologies of braid groups and groups connected with them. Among these groups are pure braid groups and generalized braid groups. We present explicit formulations of some theorems of V. I. Arnold, E. Brieskorn, D. B. Fuks, F. Cohen, V. V. Goryunov and others. The ideas of some proofs are outlined. As an application of (co)homologies of braid groups we study the Thom spectra of these groups.
\end{abstract}

Introduction. The aim of this survey is to give some ideas about (co)homology of braid groups and their generalizations. It is very well known that braids were rigorously defined by E. Artin [Art1] in 1925, although the roots of this notion are seen in the works of A. Hurwitz ([H], 1891) and R. Fricke and F. Klein ([FK], 1897). Józef Przytycki informed the author that he had seen braids in the notebooks of K.-F. Gauss. In his paper [Art2] E. Artin gives the presentation of the braid group which is very well known now. We will denote here the braid group on $n$ strings by $B r_{n}$. The group $B r_{n}$ has the generators $\sigma_{i}, i=1, \ldots, n-1$. These generators are subject to the following relations:

$$
\begin{cases}\sigma_{i} \sigma_{j}=\sigma_{j} \sigma_{i} & \text { if }|i-j|>1 \\ \sigma_{i} \sigma_{i+1} \sigma_{i}=\sigma_{i+1} \sigma_{i} \sigma_{i+1} . & \end{cases}
$$

The cohomologies of the braid groups were first studied by V. I. Arnold in the work [Arn2], published in 1970. In this paper he discusses the cohomology of the braid groups in a very broad mathematical context and displays connections of this subject with various mathematical fields. He proves three important theorems about $H^{i}\left(B r_{n}, \mathbb{Z}\right)$, namely, the theorems of finiteness, of recurrence and of stabilization (see Theorems $4.1-4.3$ below). Also he computes the cohomology groups $H^{i}\left(B r_{n}, \mathbb{Z}\right)$ for $n \leq 11$ and $i \leq 9$. Cohomologies of pure braid groups were also calculated by V. I. Arnold [Arn1]. These papers of V. I. Arnold had a great impact. His study was continued by D. B. Fuks [F1]

1991 Mathematics Subject Classification: Primary 20J05, 20F36, 20F55, 55N22.

Key words and phrases: Braid group, configuration space, homology, Coxeter group, generalized braid group, Thom spectrum, Eilenberg-MacLane spectrum.

The paper is in final form and no version of it will be published elsewhere. 
who calculated the cohomology of the braid groups mod 2. E. Brieskorn [Bri] generalized naturally the notion of the braid group for any finite Coxeter group $W$ in such a way that the classical braid group arises when we consider symmetric group as the Coxeter group of type $A_{n}$. He proves some analogues of Arnold's results for generalized braid groups and pure generalized braid groups. Independently of the works of V. I. Arnold and D. B. Fuks, the homologies of classical braid groups were studied by Fred Cohen [CF1], [CF2], [CLM] by different methods. He computed these homologies with coefficients in $\mathbb{Z}$ and in $\mathbb{Z} / p$ as modules over the Steenrod algebra. The additive structure of these cohomologies was also computed by V. F. Vainshtein [Vai] who used the methods of D. B. Fuks. Later these methods were applied by V. V. Goryunov in [G1], [G2] who expressed the cohomologies of the generalized braid groups of types $C$ and $D$ in terms of classical ones.

The cohomologies of the braid groups have the following interesting application. The canonical representation of the braid group $B r_{n}$ in the orthogonal group $O_{n}$ induces a map of the corresponding classifying spaces, and Thom spaces (details in Section 6). It was proved by Fred Cohen [CF3] and Mark Mahowald [Mah1], [Mah2] that the Thom spectrum of these spaces is the Eilenberg-MacLane spectrum of the ordinary homology with coefficients in $\mathbb{Z} / 2$.

The paper is organized as follows. In Section 1 we discuss configuration spaces whose fundamental groups are braid groups. In Section 2 we give a brief sketch of Coxeter groups and study generalized braid groups which are defined by Coxeter groups. The cohomologies of pure braid groups are given in Section 3. Various aspects of (co)homologies of classical braid groups are discussed in Section 4. Cohomologies of generalized braid groups of types $C$ and $D$ are expressed in terms of cohomologies of classical braid groups in Section 5. The study of the Thom spectra of braid groups is carried out in Section 6 .

1. Braid groups and configuration spaces. The braid group has a natural interpretation as the fundamental group of the configuration space. For our purposes it will be useful to look at braids from a very general point of view as it was done by V. Ya. Lin in [Li]. Let $Y$ be a connected topological manifold and $W$ be a finite group acting on $Y$. A point $y \in Y$ is called regular if its stabilizer $\{w \in W: w y=y\}$ is trivial, i.e. consists only of the unit of the group $W$. The set $\widetilde{Y}$ of all regular points is open. Suppose that it is connected and nonempty. The subspace $\operatorname{ORB}(Y, W)$ of the space of all orbits $\operatorname{Orb}(Y, W)$, consisting of the orbits of all regular points, is called the space of regular orbits. We have a free action of $W$ on $\widetilde{Y}$ and the projection $p: \widetilde{Y} \rightarrow \widetilde{Y} / W=O R B(Y, W)$ defines a covering. Let us consider the initial segment of the long exact sequence of this covering:

$$
1 \rightarrow \pi_{1}\left(\tilde{Y}, y_{0}\right) \stackrel{p_{*}}{\rightarrow} \pi_{1}\left(\operatorname{ORB}(Y, W), p\left(y_{0}\right)\right) \rightarrow W \rightarrow 1 .
$$

The fundamental group $\pi_{1}\left(\operatorname{ORB}(Y, W), p\left(y_{0}\right)\right)$ of the space of regular orbits will be called the braid group of the action of $W$ on $Y$ and denoted by $B r(Y, W)$. The fundamental group $\pi_{1}\left(\widetilde{Y}, y_{0}\right)$ will be called the pure braid group of the action of $W$ on $Y$ and denoted by $P(Y, W)$. The spaces $\widetilde{Y}$ and $\operatorname{ORB}(Y, W)$ are path connected, so the pair of these groups is defined uniquely up to isomorphism and it is possible to omit the base point $y_{0}$ in the notations. For any space $Y$, the symmetric group $\Sigma_{m}$ acts on Cartesian power 
$Y^{m}$ of $Y$ :

$$
w\left(y_{1}, \ldots, y_{m}\right)=\left(y_{w^{-1}(1)}, \ldots, y_{w^{-1}(m)}\right), \quad w \in \Sigma_{m}
$$

We denote by $F(Y, m)$ the space of $m$-tuples of pairwise different points in $Y$ :

$$
F(Y, m)=\left\{\left(p_{1}, \ldots, p_{m}\right) \in Y^{m}: p_{i} \neq p_{j} \text { for } i \neq j\right\} .
$$

It is the space of regular points of this action. In the case when the space $Y$ is a connected topological manifold $M$ without boundary and $\operatorname{dim} M \geq 2$, the space of regular orbits $\operatorname{ORB}\left(M^{m}, \Sigma_{m}\right)$ is open, connected and nonempty. It is called the configuration space of the manifold $M$ and is denoted by $B(M, m)$. The braid group $B r\left(M^{m}, \Sigma_{m}\right)$ is called the braid group of the manifold $M$ on $m$ strings and is denoted by $\operatorname{Br}(m, M)$. Analogously the group $P\left(M^{m}, \Sigma_{m}\right)$ is called the pure braid group of the manifold $M$ on $m$ strings and is denoted by $P(m, M)$. These definitions of braid groups were given by R. Fox and L. Neuwirth [FoN]. The classical braid group on $m$ strings $B r_{m}$ and the corresponding pure braid group $P_{m}$ are obtained in the case when the manifold $M$ is equal to the Euclidean plane $\mathbb{R}^{2}$.

Let $\left(q_{i}\right)_{i \in \mathbb{N}}$ be a fixed sequence of distinct points in the manifold $M$ and put $Q_{m}=$ $\left\{q_{1}, \ldots, q_{m}\right\}$. We use

$$
Q_{m, l}=\left(q_{l+1}, \ldots, q_{l+m}\right) \in F\left(M \backslash Q_{l}, m\right)
$$

as the standard base point of the space $F\left(M \backslash Q_{l}, m\right)$. If $k<m$ we define a projection

$$
\operatorname{proj}: F\left(M \backslash Q_{l}, m\right) \rightarrow F\left(M \backslash Q_{l}, k\right)
$$

by the formula: $\operatorname{proj}\left(p_{1}, \ldots, p_{m}\right)=\left(p_{1}, \ldots, p_{k}\right)$. The following theorems were proved by E. Fadell and L. Neuwirth $[\mathrm{FaN}]$.

THEOREM 1.1. The triple proj $: F\left(M \backslash Q_{l}, m\right) \rightarrow F\left(M \backslash Q_{l}, k\right)$ is a locally trivial fibre bundle with fibre $\operatorname{proj}^{-1} Q_{k, l}$ homeomorphic to $F\left(M \backslash Q_{k+l}, m-k\right)$.

Considering the sequence of fibrations

$$
\begin{gathered}
F\left(M \backslash Q_{m-1}, 1\right) \rightarrow F\left(M \backslash Q_{m-2}, 2\right) \rightarrow M \backslash Q_{m-2}, \\
F\left(M \backslash Q_{m-2}, 2\right) \rightarrow F\left(M \backslash Q_{m-3}, 3\right) \rightarrow M \backslash Q_{m-3}, \\
\ldots, \\
F\left(M \backslash Q_{1}, m-1\right) \rightarrow F(M, m) \rightarrow M
\end{gathered}
$$

E. Fadell and L. Neuwirth proved the following theorem.

Theorem 1.2. For any manifold $M$

$$
\pi_{i}\left(F\left(M \backslash Q_{1}, m-1\right)\right)=\oplus_{k=1}^{m-1} \pi_{i}\left(M \backslash Q_{k}\right)
$$

for $i \geq 2$. If $\pi: F(M, m) \rightarrow M$ admits a section, then

$$
\operatorname{proj}_{i} \pi_{i}(F(M, m))=\oplus_{k=0}^{m-1} \pi_{i}\left(M \backslash Q_{k}\right), i \geq 2 .
$$

Corollary 1.1. If $M$ is the Euclidean $r$-space, then

$$
\pi_{i}(F(M, m))=\oplus_{k=0}^{m-1} \pi_{i}(\underbrace{S^{r-1} \vee \ldots \vee S^{r-1}}_{k}), i \geq 2 .
$$


Corollary 1.2. If $M$ is the Euclidean 2-space, then the space $F\left(\mathbb{R}^{2}, m\right)$ is the $K\left(P_{m}, 1\right)$-space and the space $B\left(\mathbb{R}^{2}, m\right)$ is the $K\left(B r_{m}, 1\right)$-space.

2. Generalized braid groups. Let $V$ be a finite dimensional real vector space $(\operatorname{dim} V=n$ ) with Euclidean structure. We denote by $W$ a finite subgroup of $G L(V)$ generated by reflections. We use the terminology and the results of N. Bourbaki [Bo]. Let $\mathcal{M}$ be the set of hyperplanes such that $W$ is generated by orthogonal reflections with respect to $M \in \mathcal{M}$. We suppose that for any $w \in W$ and any hyperplane $M \in \mathcal{M}$ the hyperplane $w(M)$ belongs to $\mathcal{M}$. The space $V$ is divided into cells by hyperplanes of the system $\mathcal{M}$. The cells of the maximal dimension (equal to $n$ ) are called chambers. The boundary of a chamber $A$ is a subset of the union of hyperplanes. These hyperplanes are called the walls of the chamber A. The following facts are well known [Bo].

Proposition 2.1. (i) $W$ permutes the chambers of $\mathcal{M}$ transitively.

(ii) The closure $\bar{A}$ of a chamber $A$ is the fundamental domain of $W$ acting on $V$.

(iii) If $x \in V$ belongs to $\bar{A}$, its stabilizer is generated by reflections with respect to the walls of $A$ containing $x$.

Also there exists a set $I$ and a one to one correspondence of the elements of $I$ with the walls of a chamber $A$ : $i \mapsto M_{i}(A)$, which is called a canonical indexation of the walls of the chamber $A$. Then $W$ is generated by the reflections $w_{i}=w_{i}\left(M_{i}\right), i \in I$, satisfying only the following relations

$$
\left(w_{i} w_{j}\right)^{m_{i, j}}=e, i, j \in I,
$$

where the natural numbers $m_{i, j}=m_{j, i}$ form the Coxeter matrix of $W$ by which the Coxeter graph $\Gamma(W)$ of $W$ is constructed. We use the following notations of P. Deligne $[\mathrm{D}]: \operatorname{prod}(m ; x, y)$ denotes the product $x y x y \ldots$ ( $m$ factors). The generalized braid group $\operatorname{Br}(W)$ of $W[\mathrm{Br}],[\mathrm{D}]$ is defined as a group with generators $\left\{s_{i}, i \in I\right\}$ and the following relations:

$$
\operatorname{prod}\left(m_{i, j} ; s_{i}, s_{j}\right)=\operatorname{prod}\left(m_{j, i} ; s_{j}, s_{i}\right) \text {. }
$$

From this we obtain the presentation of the group $W$ if we add the relations:

$$
s_{i}^{2}=e ; \quad i \in I \text {. }
$$

We will see later in the Theorem 2.1 that this definition of a generalized braid group agrees with our general definition of a braid group of an action of a group $W$. We denote by $\tau_{W}$ the canonical map from $B r(W)$ to $W$. The classical braids on $k$ strings $B r_{k}$ are obtained by this construction if $W=A_{k}=\Sigma_{k+1}$, the symmetric group on $k+1$ symbols. In this case $m_{i, i+1}=3$, and $m_{i, j}=2$ if $j \neq i, i+1$.

Now let $J_{1}, \ldots, J_{s}$ be the sets of vertices of the connected components of the Coxeter graph of $W, W_{q}$ is the subgroup of $W$ generated by the reflections $w_{i}, i \in J_{q}$. Let $V_{q}^{0}$ be the subspace of $V$ consisting of vectors invariant under the action of $W_{q}, V_{q}$ is the orthogonal complement of $V_{q}^{0}$ in $V, V_{0}=\bigcap_{1 \leq q \leq s} V_{q}^{0}$. Then we get the following facts from Proposition 5 of ([Bo], Chapter V, Section 3.7).

Proposition 2.2. (i) The group $W$ is the direct product of the subgroups $W_{q}(1 \leq$ $q \leq s)$. 
(ii) The vector space $V$ is the direct sum of the orthogonal subspaces $V_{1}, \ldots, V_{s}, V_{0}$ invariant under the action of $W$.

If $V_{0}=0$, then the group $W$ acting on $V$ is called essential. In this case each chamber is an open simplicial cone. Let us define some ordering on all the walls $\mathcal{M}_{i}, 1 \leq i \leq n$ of a chamber $A$. The product of reflections $w_{\mathcal{M}_{1}} w_{\mathcal{M}_{2}} \ldots w_{\mathcal{M}_{n}}$ is called the Coxeter transformation defined by the ordered chamber $A$. All the Coxeter transformations are conjugate in $W$ and so, all of them have the same characteristic polynomial and the same (finite) order. This order is called the Coxeter number of the group $W$. We denote the Coxeter number of the group $W$ by $h$. Then the characteristic polynomial of a Coxeter transformation can be written in the form:

$$
f(t)=\prod_{j=1}^{n}\left(t-\exp \left(\frac{2 i \pi m_{j}}{h}\right)\right),
$$

where $m_{1}, m_{2}, \ldots, m_{n}$ are the integers such that

$$
0 \leq m_{1} \leq m_{2} \leq \ldots \leq m_{n}<h .
$$

The integers $m_{1}, m_{2}, \ldots, m_{n}$ are called the exponents of the group $W$.

The classification of irreducible (with connected Coxeter graph) Coxeter groups is well known (Theorem 1 , Chapter VI, Section 4 of [Bo]). It consists of the three infinite series: $A, C$ and $D$ and groups $E_{6}, E_{7}, E_{8}, F_{4}, G_{2}, H_{3}, H_{4}$ and $I_{2}(p)$. As an example we show here the Coxeter graphs for $A_{n}, C_{n}, D_{n}$ and $E_{8}$ :

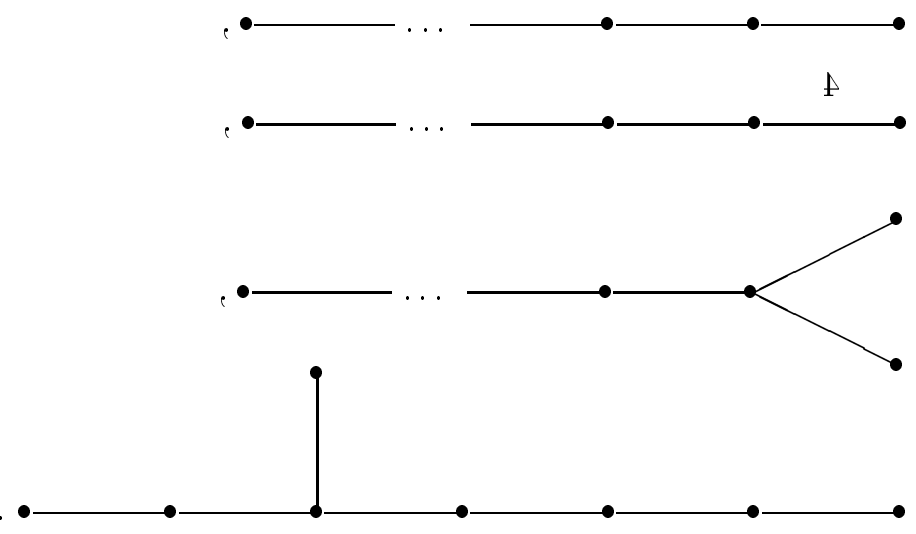

The number of vertices in these diagrams is equal to $n$, and the number $m$ over an edge means that $m_{i, j}=m$ for the pair of generators, corresponding to the points connected by the given edge.

Now let us consider the complexification $V_{C}$ of $V$ and the complexification $M_{C}$ of $M \in \mathcal{M}$. Let $Y_{W}=V_{C}-\bigcup_{M \in \mathcal{M}} M_{C}$. Then from (iii) of Proposition 2.1 we get that $W$ acts freely on $Y_{W}$. Let $X_{W}=Y_{W} / W, Y_{W}$ be a covering over $X_{W}$ corresponding to the group $W$. Let $y_{0} \in A_{0}$ be a point in some chamber $A_{0}$ and $x_{0}$ its image in $X_{W}$. We are in the situation described in Section 1 in the definition of the braid group of the action of the group $W$. This braid group is defined as the fundamental group of the space of regular orbits of the action of $W$. In our case $O R B\left(V_{C}, W\right)=X_{W}$. So, the generalized 
braid group is equal to $\pi_{1}\left(X_{W}, x_{0}\right)$. For each $j \in I$, let $\ell_{j}^{\prime}$ be the homotopy class of paths in $Y_{W}$, starting from $y_{0}$ and ending in $w_{j}\left(y_{0}\right)$, which contains a polygon line with successive vertices: $y_{0}, y_{0}+i y_{0}, w_{j}\left(y_{0}\right)+i y_{0}, w_{j}\left(y_{0}\right)$. The image $\ell_{j}$ of $\ell_{j}^{\prime}$ in $X_{W}$ is a loop with base point $x_{0}$.

THEOREM 2.1. (i) The fundamental group $\pi_{1}\left(X_{W}, x_{0}\right)$ is generated by the elements $\ell_{j}$ satisfying the following relations:

$$
\operatorname{prod}\left(m_{j, k} ; \ell_{j}, \ell_{k}\right)=\operatorname{prod}\left(m_{k, j} ; \ell_{k}, \ell_{j}\right) .
$$

(ii) The universal covering of $X_{W}$ is contractible, and so $X_{W}$ is $K(\pi ; 1)$.

Item (i) of this theorem was proved by E. Brieskorn [Bri]. Item (ii) for the groups of types $C_{n}, G_{2}$ and $I_{2}(p)$ was also proved by E. Brieskorn [Bri], similarly as E. Fadell and L. Neuwirth [FaN] proved Theorems 1.1, 1.2 and Corollary 1.2. For the types $D_{n}$ and $F_{4}$, E. Brieskorn used this method with small modifications. In the general case (ii) was proved by P. Deligne [D].

If a group $W$ is the direct product of groups $W^{\prime}$ and $W^{\prime \prime}$, then the group $\operatorname{Br}(W)$ is the direct product of the groups $\operatorname{Br}\left(W^{\prime}\right)$ and $\operatorname{Br}\left(W^{\prime \prime}\right)$. So, if a group $W$ is the same as in Proposition 2.2, then we have: $\operatorname{Br}(W)=\operatorname{Br}\left(W_{1}\right) \times \ldots \times \operatorname{Br}\left(W_{s}\right)$.

There exist pairings for symmetric and braid groups

$$
\begin{gathered}
\Sigma_{k} \times \Sigma_{l} \rightarrow \Sigma_{k+l}, \\
\mu: B r_{k} \times B r_{l} \rightarrow B r_{k+l},
\end{gathered}
$$

which commute with the maps $\tau_{j}: B r_{j} \rightarrow \Sigma_{j}$. For the braid group this pairing may be constructed by means of adding $l$ extra strings to the initial $k$. If $\sigma_{i}^{\prime}$ are the generators of $B r_{k}, \sigma_{j}^{\prime \prime}$ are the generators of $B r_{l}$, and $\sigma_{r}$ are the generators of $B r(k+l)$, then the map $\mu$ can be expressed in the form:

$$
\begin{gathered}
\mu\left(\sigma_{i}^{\prime}, e\right)=\sigma_{i}, 1 \leq i \leq k-1, \\
\mu\left(e, \sigma_{j}^{\prime \prime}\right)=\sigma_{j+k}, 1 \leq j \leq l-1 .
\end{gathered}
$$

In terms of Coxeter graphs it means that we take the vertex corresponding to $\sigma_{k}$ in the Coxeter graph $\Gamma\left(\Sigma_{k+l}\right)$ and embed $B r_{k} \times B r_{l}$ into $B r_{k+l}$ in accordance with the inclusion of the $\Gamma\left(\Sigma_{k} \times \Sigma_{l}\right)=\Gamma\left(\Sigma_{k}\right) \bigcup \Gamma\left(\Sigma_{l}\right)$ into the two components of the graph $\Gamma\left(\Sigma_{k+l}\right) \backslash \sigma_{k}$. This permits us to interpret various embeddings of products of finite Coxeter groups into a group with greater index. It is true for the corresponding generalized braid groups as well. We take away a vertex in a connected Coxeter graph and obtain a graph whose number of connected components is less than or equal to 3 . These components correspond to irreducible Coxeter groups or braid groups whose direct product is the source of this mapping. For example, we have the evident pairings:

$$
\begin{gathered}
\mu(C, A): B r\left(C_{k}\right) \times B r\left(A_{l}\right) \rightarrow B r\left(C_{k+l+1}\right), \\
\mu(D, A): B r\left(D_{k}\right) \times B r\left(A_{l}\right) \rightarrow B r\left(D_{k+l+1}\right) \text { for any } k \text { and } l,
\end{gathered}
$$

or the pairing

$$
\mu\left(A_{3}, A_{4} ; E_{8}\right): B r\left(A_{3}\right) \times B r\left(A_{4}\right) \rightarrow \operatorname{Br}\left(E_{8}\right)
$$

that corresponds to the fourth horizontal vertex of the Coxeter graph of $E_{8}$. 
Embeddings of groups (not products) can also be expressed in this language. For example, we have the embedding

$$
\alpha_{C}: \operatorname{Br}\left(A_{l-1}\right) \rightarrow \operatorname{Br}\left(C_{l}\right)
$$

and two different embeddings:

$$
\alpha_{D}: \operatorname{Br}\left(A_{l-1}\right) \rightarrow \operatorname{Br}\left(D_{l}\right)
$$

in accordance with two different vertices on one end of the Coxeter graph for $D_{l}$.

We would like to consider a generalized braid group $\operatorname{Br}\left(C_{k}\right)$. We have a relation in $\operatorname{Br}\left(C_{k}\right)$ :

$$
w_{1} w_{2} w_{1} w_{2}=w_{2} w_{1} w_{2} w_{1} .
$$

Let $B r_{1, n+1}$ be the subgroup of the braid group $B r_{n+1}$ consisting of all elements of $B r_{n+1}$ with the property, that permutations associated with them all leave the number 1 invariant. It means that the end of the first string is again at the first place. W.-L. Chow $[\mathrm{Ch}]$ found the presentation of this group with generators:

$$
\sigma_{2}, \ldots, \sigma_{n}, a_{2}, \ldots, a_{n+1},
$$

where $\sigma_{j}$ is the standard generator of the braid group $B r_{n+1}$, and the elements $a_{i}$ are given by the equality $a_{i}=\sigma_{1}^{-1} \ldots \sigma_{i-2}^{-1} \sigma_{i-1}^{2} \sigma_{i-2} \ldots \sigma_{1}, 2 \leq i \leq n+1$. The elements $\sigma_{2}, \ldots, \sigma_{n}$ generate a subgroup in $B r_{1, n+1}$ isomorphic to $B r_{n}$, and the elements $a_{2}, \ldots, a_{n+1}$ generate a normal free subgroup $F_{n}$. The following relation is fulfilled in $B r_{1, n+1}$ :

$$
\sigma_{2} a_{2} \sigma_{2} a_{2}=a_{2} \sigma_{2} a_{2} \sigma_{2}
$$

We define the homomorphism $\phi: B r\left(C_{n}\right) \rightarrow B r_{1, n+1}$ by the formulae:

$$
\begin{aligned}
& \phi\left(w_{1}\right)=a_{2}, \\
& \phi\left(w_{i}\right)=\sigma_{i}, i=2, \ldots, n,
\end{aligned}
$$

and obtain the following statement.

Proposition 2.3. The map $\phi$ defines an isomorphism

$$
\phi: B r\left(C_{n}\right) \cong B r_{1, n+1} .
$$

The claim of this proposition is evident from the geometric point of view. The space $X_{C_{n}}$ can be interpreted as a space of $n$ different pairs of points of $\mathbb{R}^{2} \backslash 0$, symmetrical with respect to zero [G1, G2]. That is the same as simply the space of $n$ different points in $\mathbb{R}^{2} \backslash 0$. The group $B r_{n+1}$ is interpreted as the fundamental group of the space $X_{A_{n}}$ of $n+1$ different points in $\mathbb{R}^{2}$. If we consider one point (say 0) to be fixed, then we get $X_{C_{n}}$. For the fundamental group of $X_{A_{n}}$ it means that the first string must have the same end as its beginning (equal to zero).

We denote by $\beta$ the homomorphism from $\operatorname{Br}\left(C_{n}\right)$ to $B r_{n}$ defined by the formulae:

$$
\begin{aligned}
& \beta\left(w_{1}\right)=e, \\
& \beta\left(w_{i}\right)=\sigma_{i-1}, \text { for } i>1 .
\end{aligned}
$$

Then we have $\beta \alpha_{C}=1_{B r_{n}}$, and $\operatorname{Br}\left(C_{n}\right)$ is isomorphic to the semidirect product of $F_{n}$ and $B r_{n}$ with the standard braid action of $B r_{n}$ on $F_{n}$ [Bi]. It is known that the 
group $C_{k}$ is isomorphic to the wreath product of the symmetric group $\Sigma_{k}=A_{k-1}$ with $\mathbb{Z} / 2: C_{k} \cong \Sigma_{k} \prec \mathbb{Z} / 2$. The pairing

$$
m_{C}: C_{k} \times C_{l} \rightarrow C_{k+l}
$$

may be defined using the pairing for the symmetric group

$$
\Sigma_{k} \times \Sigma_{l} \rightarrow \Sigma_{k+l}
$$

and the wreath product structure.

Let $w_{1}^{\prime}, \ldots, w_{k}^{\prime}$ be the generators of $\operatorname{Br}\left(C_{k}\right)$ and $w_{1}^{\prime \prime}, \ldots, w_{l}^{\prime \prime}$ be the generators of $\operatorname{Br}\left(C_{l}\right)$. Then it is possible to define the pairings $\mu_{1}(C, C)$ and $\mu_{2}(C, C)$ :

$$
\begin{gathered}
\mu_{1}(C, C): B r\left(C_{k}\right) \times B r\left(C_{l}\right) \rightarrow B r\left(C_{k+l}\right), \\
\mu_{2}(C, C): B r\left(C_{k}\right) * B r\left(C_{l}\right) \rightarrow B r\left(C_{k+l}\right)
\end{gathered}
$$

by the formulae:

$$
\begin{gathered}
\mu_{1}(C, C)\left(w_{i}^{\prime}, e\right)=w_{i}, 1 \leq i \leq k, \\
\mu_{1}(C, C)\left(e, w_{1}^{\prime \prime}\right)=w_{k+1} \ldots w_{2} w_{1} w_{2} \ldots w_{k+1}, \\
\mu_{1}(C, C)\left(e, w_{j}^{\prime \prime}\right)=w_{k+j}, 1 \leq j \leq l, \\
\mu_{2}(C, C)\left(w_{i}^{\prime}, e\right)=w_{i}, 1 \leq i \leq k, \\
\mu_{2}(C, C)\left(e, w_{1}^{\prime \prime}\right)=w_{k+1} \ldots w_{2} w_{1} w_{2}^{-1} \ldots w_{k+1}^{-1}, \\
\mu_{2}(C, C)\left(e, w_{j}^{\prime \prime}\right)=w_{k+j}, 1 \leq j \leq l .
\end{gathered}
$$

The pairing $\mu_{1}$ was first defined in [Ve] and the pairing $\mu_{2}$ was introduced by Sofia Lambropoulou in her study of links in a solid torus [La]. It is easy to check that the pairing $\mu_{1}$ is associative, that is, the following diagram is commutative:

$$
\begin{array}{ccc}
\operatorname{Br}\left(C_{k}\right) \times B r\left(C_{l}\right) \times \operatorname{Br}\left(C_{q}\right) \stackrel{\mu_{1} \times 1}{\longrightarrow} & \operatorname{Br}\left(C_{k+l}\right) \times \operatorname{Br}\left(C_{q}\right) \\
\downarrow 1 \times \mu_{1} & & \downarrow \mu_{1} \\
\operatorname{Br}\left(C_{k}\right) \times \operatorname{Br}\left(C_{l+q}\right) & \stackrel{\mu_{1}}{\longrightarrow} & \operatorname{Br}\left(C_{k+l+q}\right),
\end{array}
$$

The pairing agrees with the pairing for the Coxeter groups

$$
m_{C}: C_{k} \times C_{l} \rightarrow C_{k+l},
$$

so we have a commutative diagram

$$
\begin{array}{ccc}
\operatorname{Br}\left(C_{k}\right) \times B r\left(C_{l}\right) \stackrel{\tau_{C} \times \tau_{C}}{\longrightarrow} & C_{k} \times C_{l} \\
\downarrow \mu_{1} & & \downarrow m_{C} \\
B r\left(C_{k+l}\right) & \stackrel{\tau_{C}}{\longrightarrow} & C_{k+l} .
\end{array}
$$

It also agrees with the pairing $B r\left(C_{k}\right) \times B r_{l} \rightarrow B r\left(C_{k+l}\right)$ through the canonical inclusion $B r_{l} \rightarrow B r\left(C_{l}\right)$. It is also easy to check the commutativity of the diagram for the homomorphism $\alpha_{C}$ : 


$$
\begin{array}{ccc}
B r_{k} \times B r_{l} \stackrel{\alpha_{C} \times \alpha_{C}}{\longrightarrow} & B r\left(C_{k}\right) \times B r\left(C_{l}\right) \\
\downarrow \mu & & \downarrow \mu_{1} \\
B r_{k+l} & \stackrel{\alpha_{C}}{\longrightarrow} & B r\left(C_{k+l}\right) .
\end{array}
$$

But there is no analogous commutativity for $\beta: B r\left(C_{k}\right) \rightarrow B r_{k}$ and $\mu_{1}$. To see this let $k=2$; then $\mu(\beta, \beta)\left(e, w_{1}^{\prime \prime}\right)=\mu(e, e)=e$ and $\beta \mu_{1}\left(e, w_{1}^{\prime \prime}\right)=\beta\left(w_{3} w_{2} w_{1} w_{2} w_{3}\right)=\sigma_{2} \sigma_{1}^{2} \sigma_{2} \neq$ $e$. So the homomorphism $\beta$ does not agree with the pairings $\mu$ and $\mu_{1}$.

On the level of configuration spaces, the pairing $\mu_{1}$ for the braids of the series $C$ can be described in the following way. We map $\mathbb{R}^{2} \backslash 0$ (with $k$ different points) diffeomorphically onto the open disk of radius $k+1 / 2$ without zero $\mathcal{D}_{k+1 / 2} \backslash 0$, in such a way that the points with coordinates $(1,0), \ldots,(k, 0)$ go onto themselves, and we map $\mathbb{R}^{2} \backslash 0$ (with $l$ different points) diffeomorphically onto $\mathbb{R}^{2} \backslash \mathcal{D}_{k+1 / 2}$, in such a way that the points with coordinates $(1,0), \ldots,(l, 0)$ go onto the points $(k+1,0), \ldots,(k+l, 0)$. This map

$$
\mathbb{R}^{2} \backslash 0 \times \mathbb{R}^{2} \backslash 0 \rightarrow \mathbb{R}^{2} \backslash 0
$$

is a particular case of the map of configuration spaces, which was described by Viktor Vassiliev [Vas, p. 25]:

$$
X(k) \times X(l) \rightarrow X(k+l),
$$

where the space $X(k)$ in our notations is equal to $B(X, k)$, and the space $X$ can be presented in the form $X=Y \times \mathbb{R}$ for some other space $Y$. The map (2.1) generates the pairing of fundamental groups of configuration spaces which coincides with $\mu_{1}$. Considering the generalized braid groups of type $C$ as the subgroups of the ordinary braid groups, the pairing $\mu_{1}$ can be described as putting $k+1$ strings of the first group instead of the zero string of the second group.

Let us consider the group $B r_{k} \prec \mathbb{Z} / 2$, which can be viewed as semi-direct product of $B r_{k}$ with $\mathbb{Z} / 2 \oplus \cdots \oplus \mathbb{Z} / 2$ ( $k$ copies), where $B r_{k}$ acts on $\mathbb{Z} / 2 \oplus \cdots \oplus \mathbb{Z} / 2$ by permutations. We denote by $s_{1}$ the element $(a, e, \ldots, e) \in \mathbb{Z} / 2 \oplus \cdots \oplus \mathbb{Z} / 2$, where $a$ is a generator of $\mathbb{Z} / 2$, and we denote the standard generators of $B r_{k}$ by $s_{2}, \ldots, s_{k}$ in this context. Then we have the relation:

$$
s_{1} s_{2} s_{1} s_{2}=s_{2} s_{1} s_{2} s_{1} \text {. }
$$

We define the homomorphism

$$
\gamma: B r\left(C_{k}\right) \rightarrow B r_{k} \prec Z / 2
$$

by the formula

$$
\gamma\left(w_{i}\right)=s_{i}
$$

This homomorphism does not agree with the pairings $\left(\mu_{1}\right.$ and the pairing determined by the wreath product structure).

Now we will consider the direct limits of finite Coxeter groups. We denote by $\mathcal{W}$ the category whose objects are finite Coxeter groups, and morphisms are the inclusions $W^{\prime} \mapsto W$ corresponding to inclusions of Coxeter graphs $\Gamma^{\prime} \mapsto \Gamma$. We call by a chain a subcategory $\mathcal{E}$ of $\mathcal{W}$, which is a well ordered countable set, and such that the total number of connected components of Coxeter graphs of the elements of $\mathcal{E}$ is bounded by some natural number $N_{\mathcal{E}}$ (for a subgroup $W^{\prime}$ of $W$ we consider $\Gamma^{\prime}$ as a subgraph of $\Gamma$ ). 
We call by a limit Coxeter group $W_{\infty}$, an infinite group for which there exists a chain $\mathcal{E}$, such that $W_{\infty}$ is equal to the direct limit of $\mathcal{E}$. If we take as $\mathcal{E}$ the groups from one of the series $A, C$ or $D$, with canonical inclusions as morphisms, we obtain $A_{\infty}, C_{\infty}$ or $D_{\infty}$ as the corresponding limit Coxeter groups.

Proposition 2.4. The limit Coxeter group $W_{\infty}$ is isomorphic to a direct product of a finite number (greater or equal than one) of groups of type $A_{\infty}, C_{\infty}$ or $D_{\infty}$ and of a finite number of finite Coxeter groups.

The proof follows from the fact that $W_{\infty}$ must be infinite and its Coxeter graph is to have finitely many components.

Some pairings described above generate pairings of limit Coxeter groups and the corresponding braid groups, for example

$$
\begin{aligned}
& \mu(C, A): B r\left(C_{\infty}\right) \times B r\left(A_{\infty}\right) \rightarrow B r\left(C_{\infty}\right), \\
& \mu(D, A): B r\left(D_{\infty}\right) \times B r\left(A_{\infty}\right) \rightarrow B r\left(D_{\infty}\right) .
\end{aligned}
$$

For the general limit Coxeter group $W_{\infty}$, we may have several different pairings with $\operatorname{Br}\left(A_{\infty}\right)=B r_{\infty}$, depending on the copy of one of the infinite groups of types $A_{\infty}, C_{\infty}$ or $D_{\infty}$ for which this pairing is taken

$$
\mu(W, A): B r\left(W_{\infty}\right) \times B r\left(A_{\infty}\right) \rightarrow B r\left(W_{\infty}\right) .
$$

3. Cohomology of pure braid groups. Cohomologies of pure braid groups were first calculated by V. I. Arnold in [Arn1] using the Serre spectral sequence. We consider a somewhat more general case of configuration space for $\mathbb{R}^{n}[\mathrm{O}],[\mathrm{CT}]$. Let us consider $F\left(\mathbb{R}^{n}, 2\right)$. The map

$$
\phi: S^{n-1} \rightarrow F\left(\mathbb{R}^{n}, 2\right),
$$

described by $\phi(x)=(x,-x)$, is a $\Sigma_{2}$-equivariant homotopy equivalence. Define $A$ to be the generator of $H^{n-1}\left(F\left(\mathbb{R}^{n}, 2\right), \mathbb{Z}\right)$ which is mapped by $\phi^{*}$ to the standard generator of $H^{n-1}\left(S^{n-1}, \mathbb{Z}\right)$. For $i$ and $j$, such that $1 \leq i, j \leq m, i \neq j$, specify $\pi_{i, j}: F\left(\mathbb{R}^{n}, m\right) \rightarrow$ $F\left(\mathbb{R}^{n}, 2\right)$ by $\pi_{i, j}\left(p_{1}, \ldots, p_{m}\right)=\left(p_{i}, p_{j}\right)$. Let

$$
A_{i, j}=\pi_{i, j}^{*}(A) \in H^{n-1}\left(F\left(\mathbb{R}^{n}, m\right), \mathbb{Z}\right) .
$$

It follows that $A_{i, j}=(-1)^{n} A_{j, i}$ and $A_{i, j}^{2}=0$. For $w \in \Sigma_{m}$ we have an action $w\left(A_{i, j}\right)=$ $A_{w^{-1}(i), w^{-1}(j)}$, since $\pi_{i, j} w=\pi_{w^{-1}(i), w^{-1}(j)}$. Note also that under restriction to

$$
F\left(\mathbb{R}^{n} \backslash Q_{k}, m-k\right) \cong \pi^{-1}\left(Q_{k}\right) \subset F\left(\mathbb{R}^{n}, m\right)
$$

the classes $A_{i, j}$ with $1 \leq i, j \leq k$ go to zero, since in this case the map $\pi_{i, j}$ is constant on $\pi^{-1}\left(Q_{k}\right)$. Considering the Serre spectral sequence we have the following theorem.

THEOREM 3.1. The cohomology group $H^{*}\left(F\left(\mathbb{R}^{n} \backslash Q_{k}, m-k\right), \mathbb{Z}\right)$ is the free Abelian group with generators

$$
A_{i_{1}, j_{1}} A_{i_{2}, j_{2}} \ldots A_{i_{s}, j_{s}}
$$

where $k<j_{1}<j_{2}<\cdots<j_{s} \leq m$ and $i_{r}<j_{r}$ for $r=1, \ldots s$. 
The multiplicative structure and the $\Sigma_{m}$-algebra structure of $H^{*}\left(F\left(\mathbb{R}^{n}, m\right), \mathbb{Z}\right)$ are given by the following theorem which is proved using the $\Sigma_{3}$-action on $H^{*}\left(F\left(\mathbb{R}^{n}, 3\right), \mathbb{Z}\right)$.

TheOREM 3.2. The cohomology ring $H^{*}\left(F\left(\mathbb{R}^{n}, m\right), \mathbb{Z}\right)$ is multiplicatively generated by the square-zero elements

$$
A_{i, j} \in H^{n-1}\left(F\left(\mathbb{R}^{n}, m\right), \mathbb{Z}\right), 1 \leq i<j \leq m,
$$

subject to the only relations

$$
A_{i, k} A_{j, k}=A_{i, j} A_{j, k}-A_{i, j} A_{i, k} \text { for } i<j<k .
$$

The Poincaré series for $F\left(\mathbb{R}^{n}, m\right)$ is $\prod_{j=1}^{m-1}\left(1+j t^{n-1}\right)$.

R e m ark. In the case of $\mathbb{R}^{2}=\mathbb{C}$ the cohomology classes $A_{j, k}$ can be interpreted as the cohomology classes of differential forms

$$
\omega_{j, k}=\frac{1}{2 \pi i} \frac{d z_{j}-d z_{k}}{z_{j}-z_{k}} .
$$

The cohomologies of pure generalized braid groups were computed by E. Brieskorn [Bri] using ideas of V. I. Arnold for classical case. Let $\mathcal{V}$ be a finite-dimensional complex vector space and $H_{j} \in \mathcal{V}, j \in I$ be the finite family of complex affine hyperplanes given by linear forms $l_{j}$. E. Brieskorn proves the following theorem.

THEOREM 3.3. The cohomology classes, corresponding to holomorphic differential forms

$$
\omega_{j}=\frac{1}{2 \pi i} \frac{d l_{j}}{l_{j}}
$$

generate the cohomology ring $H^{*}\left(\mathcal{V} \backslash \cup_{j \in I} H_{j}, \mathbb{Z}\right)$. Moreover, this ring is isomorphic to the $\mathbb{Z}$-subalgebra generated by the forms $\omega_{j}$ in the algebra of meromorphic forms on $\mathcal{V}$.

The cohomologies of generalized pure braid groups are described by the following theorem.

TheOREM 3.4. (i) The cohomology group of the pure braid group $P(W)$ with coeffcients in the ring of the integers $H^{k}(P(W), \mathbb{Z})$ is a free abelian group, and its rank is equal to the number of elements $w \in W$ of the length $l(w)=k$, where $l$ is the length considered with respect to the system of generators consisting of all reflections of the group $W$.

(ii) The Poincaré series for $H^{*}(P(W), \mathbb{Z})$ is

$$
\prod_{j=1}^{n}\left(1+m_{j} t\right)
$$

where $m_{j}$ are the exponents of the group $W$.

(iii) The multiplicative structure of $H^{*}(P(W), \mathbb{Z})$ coincides with the structure of algebra, generated by 1-forms described in the previous theorem.

4. Homology of braid groups. The cohomologies of the classical braid groups were first studied by V. I. Arnold in the article [Arn2]. To investigate $H^{*}\left(B r_{n}, \mathbb{Z}\right)$ he interprets 
the space $K\left(B r_{n}, 1\right) \cong B\left(\mathbb{R}^{2}, n\right)$ as the space of complex polynomials of degree $n$ without multiple roots with the first coefficient equal to 1 :

$$
P_{n}(t)=t^{n}+z_{1} t^{n-1}+\cdots+z_{n-1} t+z_{n} .
$$

More precisely let us consider the "Viète map" from $\mathbb{C}^{n}$, which we denote at this place by $\mathbb{C}_{(\lambda)}^{n}$, to $\mathbb{C}^{n}$, which we denote by $\mathbb{C}_{(z)}^{n}$ to distinguish between the domain and the image:

$$
p: \mathbb{C}_{(\lambda)}^{n} \rightarrow \mathbb{C}_{(z)}^{n}
$$

It maps a point $\left(\lambda_{1}, \ldots, \lambda_{n}\right) \in \mathbb{C}_{(\lambda)}^{n}$ to the polynomial $P_{n}(t)=t^{n}+z_{1} t^{n-1}+\cdots+z_{n-1} t+z_{n}$, which has the roots $\lambda_{1}, \ldots, \lambda_{n}$ (counted with multiplicity). The space $\mathbb{C}_{(z)}^{n}$ is interpreted as a space of orbits $\operatorname{Orb}\left(\mathbb{C}^{n}, \Sigma_{n}\right)$ of the canonical action of symmetric group $\Sigma_{n}$ on $\mathbb{C}^{n}$ by taking values of symmetrical polynomials in $\lambda_{1}, \ldots, \lambda_{n}$. The standard basis here consists of the basic symmetric polynomials:

$$
z_{k}(\lambda)=(-1)^{k} \sum_{i_{1}<\cdots<i_{k}} \lambda_{i_{1}} \ldots \lambda_{i_{k}}, 1 \leq k \leq n
$$

So,

$$
\mathbb{C}_{(z)}^{n} \cong \operatorname{Orb}\left(\mathbb{C}^{n}, \Sigma_{n}\right)
$$

and $\operatorname{ORB}\left(\mathbb{C}^{n}, \Sigma_{n}\right)$ is the space of regular orbits of this action. We see now that the map $p$ from (4.2) defines a homeomorphism

$$
p: O R B\left(\mathbb{C}^{n}, \Sigma_{n}\right) \rightarrow \mathbb{C}_{(z)}^{n} \backslash \Delta_{n}
$$

where $\Delta_{n}$ is the discriminant surface of the polynomial (4.1): the subspace of $\mathbb{C}^{n}$ where the discriminant $\Delta_{n}(z)$ is equal to zero,

$$
\Delta(z)=\Delta(p(\lambda))=\prod_{i \neq j}\left(\lambda_{i}-\lambda_{j}\right)=(-1)^{\frac{n(n-1)}{2}} \prod_{i<j}\left(\lambda_{i}-\lambda_{j}\right)^{2} .
$$

Further, V. I. Arnold considers the canonical inclusion of the space $\mathbb{C}_{(z)}^{n} \backslash \Delta$ into the sphere $S^{2 n}$ :

$$
\mathbb{C}_{(z)}^{n} \backslash \Delta \rightarrow S^{2 n}
$$

The complement of $\mathbb{C}_{(z)}^{n} \backslash \Delta$ in $S^{2 n}$ is equivalent to the one-point compactification $\Delta_{n}^{*}$ of $\Delta_{n}$. Then using the Alexander duality one has

$$
H^{i}\left(B r_{n} ; \mathbb{Z}\right)=H^{i}\left(\mathbb{C}_{(z)}^{n} \backslash \Delta_{n} ; \mathbb{Z}\right) \cong H_{2 n-i}\left(S^{2 n}, \Delta_{n}^{*} ; \mathbb{Z}\right) \cong \widetilde{H}_{2 n-i+1}\left(\Delta_{n}^{*} ; \mathbb{Z}\right),
$$

where the tilde denotes the reduced homology. Arnold proves the following facts about cohomology of the braid groups.

THEOREM 4.1 (of finiteness). The cohomology groups of the braid groups are finite except

$$
H^{0}\left(B r_{n}, \mathbb{Z}\right) \cong \mathbb{Z}, H^{1}\left(B r_{n}, \mathbb{Z}\right) \cong \mathbb{Z}, n \geq 2
$$

Also we have

$$
H^{i}\left(B r_{n}, \mathbb{Z}\right)=0 \text { if } i \geq n
$$

TheOREm 4.2 (of recurrence). All the cohomology groups of the braid group on odd number of strings are the same as the cohomology groups of the braid group on previous 
even number of strings:

$$
H^{i}\left(B r_{2 n+1}, \mathbb{Z}\right)=H^{i}\left(B r_{2 n}, \mathbb{Z}\right) .
$$

TheOREM 4.3 (of stabilization). By the increasing of $n$ the cohomology group $H^{i}\left(B r_{n}, \mathbb{Z}\right)$ of the braid group stabilizes:

$$
H^{i}\left(B r_{n}, \mathbb{Z}\right)=H^{i}\left(B r_{2 i-2}, \mathbb{Z}\right) \text { if } n \geq 2 i-2 .
$$

Remark. The isomorphism $H^{1}\left(B r_{n}, \mathbb{Z}\right) \cong \mathbb{Z}$ follows from the fact that the abelianization of $B r_{n}$ is equal to $\mathbb{Z}$. Really, we can define a homomorphism from braids to integers by taking the sum of exponents of the entries of the generators $\sigma_{i}$ in the expression of any element of the group in terms of these canonical generators:

$$
\operatorname{deg}: B r_{n} \rightarrow \mathbb{Z}, \operatorname{deg}(b)=\sum_{j} m_{j}, \text { where } b=\left(\sigma_{i_{1}}\right)^{m_{1}} \ldots\left(\sigma_{i_{k}}\right)^{m_{k}} .
$$

It is easy to prove that the kernel of $\mathrm{deg}$ is generated by commutators. Equality (4.3) follows from the fact that the space $C_{(z)}^{n} \backslash \Delta_{n}$ is a direct product of $\mathbb{C}$ and a Stein manifold.

The groups $H^{i}\left(B r_{n}, \mathbb{Z}\right)$ for $n \leq 11$ and $i \leq 9$ were also computed by V. I. Arnold.

The study of the cohomology of braid groups was continued by D. B. Fuks [F1], who calculated the cohomologies of the braid groups mod 2. For simplicity let us denote by $\Gamma_{n}$ the configuration space $B\left(\mathbb{R}^{2}, n\right)=B(\mathbb{C}, n)=F(\mathbb{C}, n) / \Sigma_{n}$. Let $\Gamma_{n}^{*}$ be the one-point compactification of $\Gamma_{n}$. Using the Poincaré duality one has:

$$
H^{k}\left(\Gamma_{n}, \mathbb{Z} / 2\right) \cong \widetilde{H}_{2 n-k}\left(\Gamma_{n}^{*}, \mathbb{Z} / 2\right) .
$$

Here $\widetilde{H}_{2 n-k}\left(\Gamma_{n}^{*}, \mathbb{Z} / 2\right)$ denotes the reduced homology group of the space $\Gamma_{n}^{*}$. To investigate the group $H_{j}\left(\Gamma_{n}^{*}, \mathbb{Z} / 2\right)$ some natural cellular decomposition of the space $\Gamma_{n}^{*}$ is constructed. Using this decomposition all the groups $H^{i}\left(B r_{n}, \mathbb{Z} / 2\right)$ are computed, the multiplicative structure of the ring $H^{*}\left(B r_{n}, \mathbb{Z} / 2\right)$ and connections with the cohomology $H^{*}\left(B O_{n}, \mathbb{Z} / 2\right)$ are described. The Hopf algebra structure of the cohomology of the infinite braid group $H^{*}\left(B r_{\infty}, \mathbb{Z} / 2\right)$ arising from the canonical pairing:

$$
B r_{n} \times B r_{m} \rightarrow B r_{n+m}
$$

is also considered in the paper [F1]. Although the results are formulated there in the language of cohomology it is more convenient to translate them to homology. Then the main results will be the following

THEOREM 4.4. The homology of the infinite braid group with coefficients in $\mathbb{Z} / 2$ is isomorphic as a Hopf algebra to the polynomial algebra on infinitely many generators $a_{i}, i=1,2, \ldots ; \operatorname{deg} a_{i}=2^{i}-1$ :

$$
H_{*}\left(B r_{\infty}, \mathbb{Z} / 2\right) \cong \mathbb{Z} / 2\left[a_{1}, a_{2}, \ldots, a_{i}, \ldots\right],
$$

with the coproduct given by the formula:

$$
\Delta\left(a_{i}\right)=1 \otimes a_{i}+a_{i} \otimes 1 .
$$

THEOREM 4.5. The canonical inclusion $\mathrm{Br}_{n} \rightarrow B r_{\infty}$ induces a monomorphism in homology with coefficients in $\mathbb{Z} / 2$. Its image is the subcoalgebra of the polynomial algebra 
$(\mathbb{Z} / 2)\left[a_{1}, a_{2}, \ldots, a_{i}, \ldots\right]$ with $\mathbb{Z} / 2$-basis consisting of monomials

$$
a_{1}^{k_{1}} \ldots a_{l}^{k_{l}}, \text { such that } \sum_{i} k_{i} 2^{i} \leq n
$$

TheOrem 4.6. The canonical homomorphism $B r_{n} \rightarrow B O_{n}, 1 \leq n \leq \infty$ induces a monomorphism (of Hopf algebras if $n=\infty$ )

$$
H_{*}\left(B r_{n}, \mathbb{Z} / 2\right) \rightarrow H_{*}\left(B O_{n}, \mathbb{Z} / 2\right) .
$$

It was noticed that configuration spaces have connections with the theory of iterated loop spaces. Peter May [May] proved that the space $F\left(\mathbb{R}^{n}, m\right)$ is $\Sigma_{m}$-equivariantly homotopy equivalent to the space $\mathcal{C}_{n}(m)$ of the little cube operad. This operad plays a key role in the recognition principle for $n$-fold loop spaces. The homologies of configuration spaces were used in the construction of Araki-Kudo-Dyer-Lashof operations in the homology of $n$-fold loop spaces [CLM]. Independently of the works of V. I. Arnold and D. B. Fuks these homologies were studied by Fred Cohen [CF1], [CF2], [CLM]. He uses the spectral sequence of a covering which has initial term

$$
E_{2}^{*, *} \cong H^{*}\left(\Sigma_{m} ; H^{*}\left(F\left(\mathbb{R}^{n}, m\right) ; \mathbb{Z} / p\right)\right),
$$

and converges to $H^{*}\left(B\left(\mathbb{R}^{n}, m\right) ; \mathbb{Z} / p\right)$. The cohomologies of symmetric group $\Sigma_{m}$ are considered with the nontrivial action of $\Sigma_{m}$ on the coefficient group $H^{*}\left(F\left(\mathbb{R}^{n}, m\right) ; \mathbb{Z} / p\right)$, which was described in the Theorem 3.2. The key observation for the calculations is the following theorem.

THEOREM 4.7 (Vanishing theorem). In the spectral sequence $\left\{E_{r}\right\}, E_{2}^{s, t}=0$ for $s>0$ and $0<t<(n-1)(p-1)$.

F. Cohen calculates the homology of $B\left(\mathbb{R}^{n}, m\right)$ with coefficients in $\mathbb{Z} / 2$ (as in Theorems 4.4 and 4.5 ) and with coefficients in $\mathbb{Z} / p, p>2$.

THEOREM 4.8. The homology of the infinite braid group with coefficients in $\mathbb{Z} / p, p>2$ as a Hopf algebra is isomorphic to the tensor product of exterior and polynomial algebras:

$$
\begin{aligned}
E\left(a_{1}, \ldots a_{i}, \ldots\right) \otimes \mathbb{Z} / p\left[b_{1}, \ldots, b_{j}, \ldots\right], & \\
& i=1,2, \ldots ; j=1,2, \ldots ; \operatorname{deg} a_{i}=2 p^{i-1}-1, \operatorname{deg} b_{j}=2 p^{j}-2,
\end{aligned}
$$

with the coproduct given by the formulae:

$$
\Delta\left(a_{i}\right)=1 \otimes a_{i}+a_{i} \otimes 1, \quad \Delta\left(b_{j}\right)=1 \otimes b_{j}+b_{j} \otimes 1 .
$$

THEOREM 4.9. The canonical inclusion $B r_{n} \rightarrow B r_{\infty}$ induces a monomorphism in homology with coefficients in $\mathbb{Z} / p, p>2$. Its image is the subcoalgebra of the tensor product $E\left(a_{1}, \ldots a_{j}, \ldots\right) \otimes \mathbb{Z} / p\left[b_{1}, \ldots, b_{r}, \ldots\right]$ with $\mathbb{Z} / p$-basis consisting of monomials

$$
a_{1}^{\epsilon_{1}} \ldots a_{l}^{\epsilon_{l}} b_{1}^{k_{1}} \ldots b_{s}^{k_{s}}, \text { where } \epsilon_{i}=0,1 ; \text { and } 2\left(\sum_{i} \epsilon_{i} p^{i-1}+\sum_{j} k_{j} p^{j}\right) \leq n .
$$

F. Cohen describes also the action of the Steenrod algebra $\mathcal{A}$ [CF3]. We would like to consider his results in the language of homology and coaction $\psi$ of the dual of the Steenrod algebra $\mathcal{A}_{*}$. Recall that, for $p=2, \mathcal{A}_{*}$ as an algebra is isomorphic to the polynomial algebra $\mathbb{Z} / 2\left[\xi_{1}, \ldots, \xi_{k}, \ldots\right]$, deg $\xi_{k}=2^{k}-1$, and for $p>2$ it is isomorphic to the tensor 
product of exterior and polynomial algebras $E\left(\tau_{0}, \tau_{1}, \ldots, \tau_{l}, \ldots\right) \otimes \mathbb{Z} / p\left[\xi_{1}, \ldots, \xi_{k}, \ldots\right]$, $\operatorname{deg} \xi_{k}=2\left(p^{k}-1\right), \operatorname{deg} \tau_{l}=2 p^{l}-1$. The coproduct $\psi$ is given by the formulae

$$
\psi\left(\xi_{k}\right)=\sum_{i+j=k} \xi_{j}^{p^{i}} \otimes \xi_{i}, \quad \psi\left(\tau_{l}\right)=\tau_{l} \otimes 1+\sum_{i+j=l} \xi_{j}^{p^{i}} \otimes \tau_{i} .
$$

Theorem 4.10. The coaction of the dual of the Steenrod algebra $\mathcal{A}_{*}$ on the homology of the braid groups $B r_{m}, 1 \leq m \leq \infty$ is given, for $p=2$, by the formula:

and for $p>2$ by the formulae

$$
\psi\left(a_{j}\right)= \begin{cases}1 \otimes a_{j}, & \text { if } j=1, \\ 1 \otimes a_{j}+\xi_{1} \otimes a_{j-1}^{2} & \text { if } j \geq 2,\end{cases}
$$

$$
\begin{aligned}
& \psi\left(a_{j}\right)= \begin{cases}1 \otimes a_{j}, & \text { if } j=1, \\
1 \otimes a_{j}+\tau_{0} \otimes b_{j-1}, & \text { if } j \geq 2,\end{cases} \\
& \psi\left(b_{j}\right)= \begin{cases}1 \otimes b_{j}, & \text { if } j=1, \\
1 \otimes b_{j}-\xi_{1} \otimes b_{j-1}^{p}, & \text { if } j \geq 2 .\end{cases}
\end{aligned}
$$

Let $\Omega_{0}^{2} S^{2}$ be the connected component of the trivial loop in the double loop space $\Omega^{2} S^{2}$. G. Segal [Se] established the following connection between the infinite braid group and iterated loop spaces.

THEOREM 4.11. There exists a map $K\left(B r_{\infty}, 1\right) \rightarrow \Omega_{0}^{2} S^{2}$, that induces an isomorphism in homology for any group of coefficients $G$ (with trivial action of $B r_{\infty}$ on $G$ ):

$$
H_{*}\left(B r_{\infty}, G\right) \cong H_{*}\left(\Omega_{0}^{2} S^{2}, G\right) .
$$

The classical Hopf fibration $S^{3} \rightarrow S^{2}$ induces isomorphism in homology $H_{*}\left(\Omega^{2} S^{3}, G\right)$ $\cong H_{*}\left(\Omega_{0}^{2} S^{2}, G\right)$. This allows us to consider the spaces $\Omega^{2} S^{3}$ and $\Omega_{0}^{2} S^{2}$ as a plus-construction for $K\left(B r_{\infty}, 1\right)$. We can also use the computations of homologies of $\Omega^{n} S^{n+1}$ by E. Dyer and R. Lashof [DL] and by Fred Cohen [CLM, p. 227]. It follows from these calculations that $H_{*}\left(\Omega^{n} S^{n+1}, \mathbb{Z} / p\right)$ is isomorphic to the polynomial algebra over $\mathbb{Z} / p$ on generators $Q_{1}^{k_{1}} \ldots Q_{n-1}^{k_{n-1}} a_{1}$, where $Q_{i}$ are the Araki-Kudo-Dyer-Lashof operations, which act in homology of iterated loop spaces [CLM], and $a_{1}$ is the image of the generator of $H_{1}\left(S^{1}, \mathbb{Z} / p\right)$ under the map $H_{*}\left(S^{1}, \mathbb{Z} / p\right) \rightarrow H_{*}\left(\Omega^{n} S^{n+1}, \mathbb{Z} / p\right)$, induced by the canonical inclusion $S^{1} \rightarrow \Omega^{n} S^{n+1}$. This gives us another proof of Theorems 4.4 and 4.8. The generators $a_{j}, j>1$, can be considered as $Q_{1}^{j-1} a_{1}$ and $b_{j}$ as $\beta a_{j+1}$. The coaction of the dual of the Steenrod algebra, described in Theorem 4.10, may be obtained from the Theorem 4.11 with the help of Nishida relations [CLM], which relate the action of Steenrod operations and Araki-Kudo-Dyer-Lashof operations.

F. Cohen uses the Bockstein spectral sequence to calculate the integral homology of the braid groups [CLM].

TheOREM 4.12. The $p$-torsion in $H_{*}\left(B r_{n}, \mathbb{Z}\right), n \leq \infty$ is all of order $p$. The $p$-torsion of $H_{*}\left(B r_{\infty}, \mathbb{Z}\right)$ in degrees strictly greater than one is isomorphic to the following:

(i) If $p=2$, to the polynomial algebra generated by $a_{1}$ and $a_{j}^{2}, j>1$.

(ii) If $p>2$, to the tensor product of exterior algebra generated by $a_{1}$, and polynomial algebra generated by $b_{j}$. 
ThEOREM 4.13. The canonical inclusion $B r_{n} \rightarrow B r_{\infty}$ induces a monomorphism in homology with coefficients in $\mathbb{Z}$. Its 2-torsion image in degrees strictly greater than 1 has the $\mathbb{Z} / 2$-basis consisting of monomials

$$
a_{1}^{k_{1}} \ldots a_{l}^{k_{l}}, \text { such that } k_{i} \equiv 0 \bmod 2 \text { for } i>1 \text { and } \sum_{i} k_{i} 2^{i} \leq n,
$$

and its $p$-torsion image, $p>2$, in degrees strictly greater than 1 has the $\mathbb{Z} / p$-basis consisting of monomials

$$
a_{1}^{\epsilon_{1}} b_{1}^{k_{1}} \ldots b_{s}^{k_{s}}, \text { where } \epsilon_{1}=0,1 ; \text { and } 2\left(\epsilon_{1}+\sum_{j} k_{j} p^{j}\right) \leq n .
$$

The methods of D. B. Fuks were applied by F. V. Vainshtein [Vai] for calculation of the cohomologies of the braid groups with coefficients in $\mathbb{Z} / p$ and $\mathbb{Z}$. As a result he obtained a complete information about the additive structure of these cohomologies and about the action of the Bockstein homomorphism.

We call the Coxeter representation of the symmetric group $\Sigma_{n}$, the representation

$$
\Sigma_{n} \rightarrow G L_{n}(\mathbb{Z}),
$$

corresponding to the permutations of the basic vectors in $\mathbb{Z}^{n}$. The restriction to the hyperplane in $\mathbb{Z}^{n}$, given by the formula $\sum x_{i}=0$, is called the reduced Coxeter representation. These representations define the structures of $\Sigma_{n}$-modules on $\mathbb{Z}^{n}$ and $\mathbb{Z}^{n-1}$. With the help of the canonical map $B r_{n} \rightarrow \Sigma_{n}, \mathbb{Z}^{n}$ and $\mathbb{Z}^{n-1}$ become modules over $B r_{n}$. We denote these modules over $B r_{n}$ by $K_{n}$ and $\widetilde{K}_{n}$. The following theorem was proved by V. V. Vassiliev [Vas].

THEOREM 4.14. The cohomologies of the braid group with coefficients in the Coxeter representation and reduced Coxeter representation are given by the formulae:

$$
\begin{aligned}
H^{q}\left(B r_{n} ; K_{n}\right) & =\bigoplus_{i=0}^{n-1} H^{q-i}\left(B r_{n-1-i} ; \mathbb{Z}\right), n \geq 2, \\
H^{q}\left(B r_{n} ; \widetilde{K}_{n}\right) & =\bigoplus_{i=1}^{n-1} H^{q-i}\left(B r_{n-i} ; \mathbb{Z}\right), n \geq 2
\end{aligned}
$$

where we put formally $B r_{0}=\{e\}$, the group consisting of a single element.

Proof. We consider the first formula now. The module $K_{n}$ is isomorphic to the module $\operatorname{Coind}_{B r_{1, n}}^{B r_{n}} \mathbb{Z}$, coinduced from the trivial module $\mathbb{Z}$ over $B r_{1, n}$, where the subgroup $B r_{1, n}$ was defined in Section 2. So by Shapiro's Lemma [Bro, III.62] we have: $H^{*}\left(B r_{n}, K_{n}\right) \cong H^{*}\left(B r_{1, n}, \mathbb{Z}\right)$. We prove the first isomorphism by induction. For $n=2$, $B r_{1,2}$ is equal to $\mathbb{Z}$ and so, its cohomologies are the same as of the circle. The formula is true. Let $n>2$. Consider the homomorphism $\beta: B r_{1, n} \rightarrow B r_{n-1}$, defined in Section 2, and the Serre-Hochschild spectral sequence for $\beta$. As it was also described in Section 2, $\operatorname{Ker} \beta$ is the free subgroup $F_{n-1}$ of $B r_{n}$ generated by braids $a_{2}, \ldots, a_{n}$. The initial term of the Serre-Hochschild spectral sequence $E_{2}^{p, q}$ is isomorphic to: $H^{p}\left(B r_{n-1}, H^{q}\left(F_{n-1}, \mathbb{Z}\right)\right)$. There are only two first nonzero lines in this spectral sequence, because

$$
H^{0}\left(F_{n-1}, \mathbb{Z}\right) \cong \mathbb{Z}, H^{1}\left(F_{n-1}, \mathbb{Z}\right) \cong \mathbb{Z}^{n-1}, H^{q}\left(F_{n-1}, \mathbb{Z}\right) \cong 0 \text { for } q>1 .
$$


We recall that $B r_{1, n}$ is isomorphic to the semidirect product of $F_{n-1}$ and $B r_{n-1}$ with the standard braid action of $B r_{n-1}$ on $F_{n-1}$. This action on $H^{0}\left(F_{n-1}, \mathbb{Z}\right) \cong \mathbb{Z}$ generates the trivial $\mathbb{Z} B r_{n-1}$-module structure and on $H^{1}\left(F_{n-1}, \mathbb{Z}\right) \cong \mathbb{Z}^{n-1}$ it generates the structure of module $K_{n-1}$. This ends the induction step. The isomorphism for the reduced Coxeter representation follows from the exact sequence $0 \rightarrow \widetilde{K}_{n} \rightarrow K_{n} \rightarrow \mathbb{Z} \rightarrow 0$.

5. Cohomology of generalized braid groups. The methods of D. B. Fuks and V. F. Vainshtein were applied by V. V. Goryunov in [G1], [G2] to calculations of the cohomologies of the generalized braid groups of types $C$ and $D$. The configuration space $X_{C_{n}}$ for the braid groups of type $C$ was described in Section 2 as the space of $n$ different pairs of points of $\mathbb{C} \backslash 0$, symmetric with respect to zero, which is the same as simply the space of $n$ different points in $\mathbb{C} \backslash 0$. The configuration space $X_{D_{n}}$ for the braid groups of type $D$ can be described in the following way. Let us consider the geometrically distinct pairs of points in $\mathbb{C}$, symmetric with respect to zero. The degenerate case when the pair consists of one point, equal to zero, is included. Then we suppose that each nondegenerate pair is considered with different signs (plus or minus) of points. The involution $\nu$ acts on nondegenerate pairs by changing the signs and is identical on the degenerate pair. We call the two unordered sets of $n$ distinct pairs of points $\left\{p_{1}, \ldots, p_{n}\right\}$ and $\left\{q_{1}, \ldots, q_{n}\right\}$ equivalent if $q_{i}=\nu^{\epsilon_{i}}\left(p_{i}\right), \epsilon_{i}=0,1$, such that $\sum_{i=1}^{i=n} \epsilon_{i}$ is even. The space $X_{D_{n}}$ is the factor space of $n$-tuples of geometrically different pairs in $\mathbb{C}$ with respect to the equivalence relation just described.

The space $X_{A_{n-1}}=B(\mathbb{C}, n)$ was interpreted in Section 4 as the space of the polynomials, over the complex numbers, of degree $n$ without multiple roots, and with the first coefficient equal to 1 (4.1). Analogously it is possible to interpret the space $X_{C_{n}}$ as the space of polynomials over $\mathbb{C}$ of the form (4.1) without multiple and zero roots. Let $\Gamma_{n}$ be one of the spaces $X_{C_{n}}$ or $X_{D_{n}}$, and $\Gamma_{n}^{*}$ its one point compactification. The same way as D. B. Fuks, V. V. Goryunov uses the Poincaré duality

$$
H^{k}\left(\Gamma_{n}, \mathbb{Z}\right) \cong \widetilde{H}_{2 n-k}\left(\Gamma_{n}^{*}, \mathbb{Z}\right)
$$

for the calculations of the cohomology of the generalized braid groups of types $C$ and $D$. He constructs the cellular subdivision of the space $\Gamma_{n}^{*}$ and proves the following theorems.

THEOREM 5.1. The cohomologies of the infinite generalized braid groups of types $C$ and $D$ with coefficients in $\mathbb{Z}$ are expressed in terms of the cohomologies of classical ones in the following way:

$$
\begin{gathered}
H^{q}\left(B r\left(C_{\infty}\right) ; \mathbb{Z}\right)=\bigoplus_{i=0}^{q} H^{q-i}\left(B r_{\infty} ; \mathbb{Z}\right), \\
H^{q}\left(B r\left(D_{\infty}\right) ; \mathbb{Z}\right)=H^{q}\left(B r_{\infty} ; \mathbb{Z}\right) \oplus\left[\bigoplus_{i=0}^{\infty} H^{q-2 i-3}\left(B r_{\infty} ; \mathbb{Z} / 2\right)\right] .
\end{gathered}
$$

We denote by $\gamma_{n}$ the canonical inclusion

$$
\gamma_{n}: B r_{n-1} \rightarrow B r_{n}
$$


and by $\gamma_{n}^{q}$ the map induced by $\gamma_{n}$ in cohomology:

$$
\gamma_{n}^{q}: H^{q}\left(B r_{n}, \mathbb{Z}\right) \rightarrow H^{q}\left(B r_{n-1}, \mathbb{Z}\right) .
$$

Ker $\gamma_{n}^{q}$ denotes as usual the kernel of this map.

THEOREM 5.2. The cohomologies of the finite generalized braid groups of types $C$ and $D$ with coefficients in $\mathbb{Z}$ are expressed in terms of the cohomologies of classical ones in the following way:

$$
\begin{gathered}
H^{q}\left(B r\left(C_{n}\right) ; \mathbb{Z}\right)=\bigoplus_{i=0}^{n} H^{q-i}\left(B r_{n-i} ; \mathbb{Z}\right), n \geq 2, \\
H^{q}\left(\operatorname{Br}\left(D_{n}\right) ; \mathbb{Z}\right)=H^{q}\left(B r_{n} ; \mathbb{Z}\right) \oplus\left[\bigoplus_{i=0}^{\infty} \operatorname{Ker} \gamma_{n-2 i}^{q-2 i}\right] \oplus\left[\bigoplus_{j=0}^{\infty} H^{q-2 j-3}\left(B r_{n-2 j-3} ; \mathbb{Z} / 2\right)\right], n \geq 3,
\end{gathered}
$$

where we put formally $B r_{0}=\{e\}$, the group consisting of a single element.

The formula for the cohomologies of $B r\left(C_{n}\right)$ was proved in Section 4 (Theorem 4.14), because

$$
H^{q}\left(B r\left(C_{n}\right) ; \mathbb{Z}\right)=H^{q}\left(B r_{1, n+1} ; \mathbb{Z}\right)=H^{q}\left(B r_{n} ; \widetilde{K}_{n}\right) .
$$

Corollary 5.1 (Theorem of stabilization). With the increasing of $n$ the cohomology groups of the generalized braid groups of types $C$ and $D$ stabilize:

$$
\begin{aligned}
& H^{i}\left(B r\left(C_{n}\right), \mathbb{Z}\right)=H^{i}\left(B r\left(C_{2 i-2}\right), \mathbb{Z}\right) \quad \text { if } n \geq 2 i-2, \\
& H^{i}\left(\operatorname{Br}\left(D_{n}\right), \mathbb{Z}\right)=H^{i}\left(\operatorname{Br}\left(D_{2 i-1}\right), \mathbb{Z}\right) \text { if } n \geq 2 i-1 .
\end{aligned}
$$

The analogues of G. Segal's theorem about the plus-construction for classifying space of infinite braid group (Theorem 4.11) were discovered by D. B. Fuks [F2]: the plusconstruction of $K\left(B r\left(C_{\infty}\right), 1\right)$ is equal to $\Omega^{2} S^{3} \times \Omega S^{2}$ and the plus-construction of the space $K\left(\operatorname{Br}\left(D_{\infty}\right), 1\right)$ is equal to $\Omega^{2} S^{3} \times F$, where $F$ is a homotopy fibre of a map of degree 2 from $S^{3}$ to $S^{3}$.

6. Thom spectra for Coxeter and braid groups. From the definition of a finite Coxeter group $W$ we have the inclusion into the orthogonal group $O(n)$, acting on the $n$-dimensional real vector space $V$ with Euclidean structure:

$$
\nu_{W}: W \rightarrow O(n),
$$

which can be involved into the following commutative diagram:

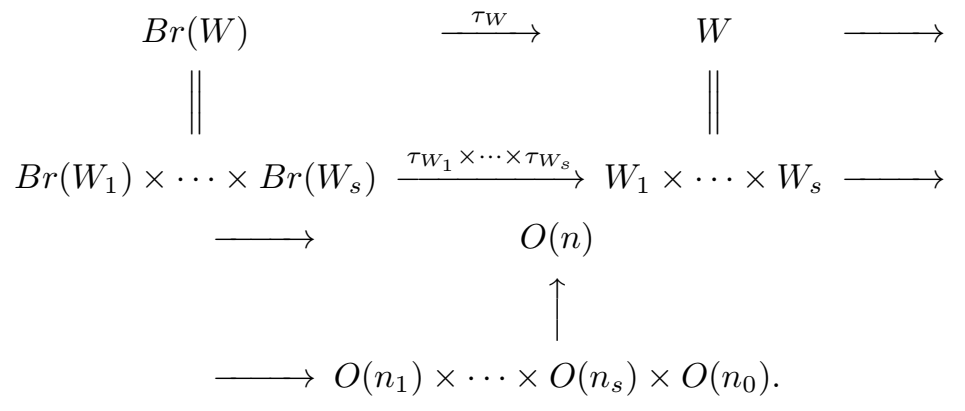


This commutative diagram generates the commutative diagram of the classifying spaces:

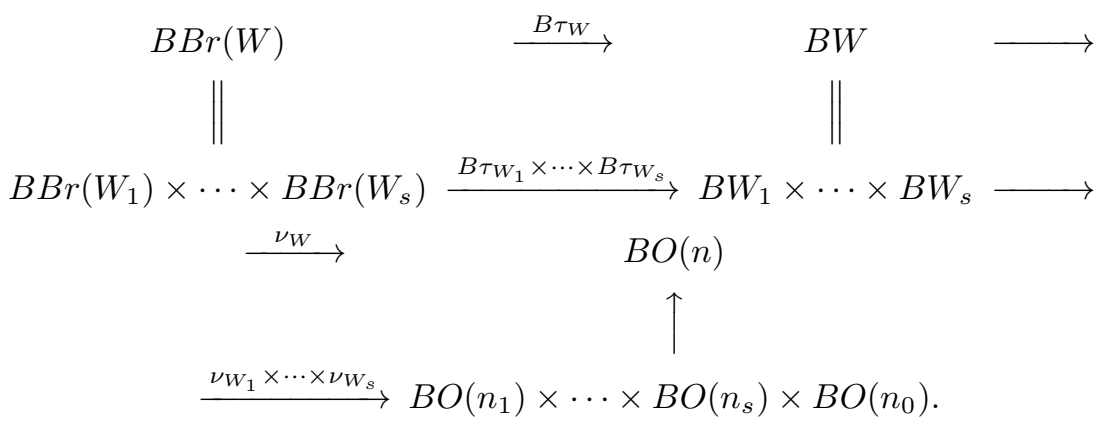

This commutative diagram generates in its turn the commutative diagram of the Thom spaces:

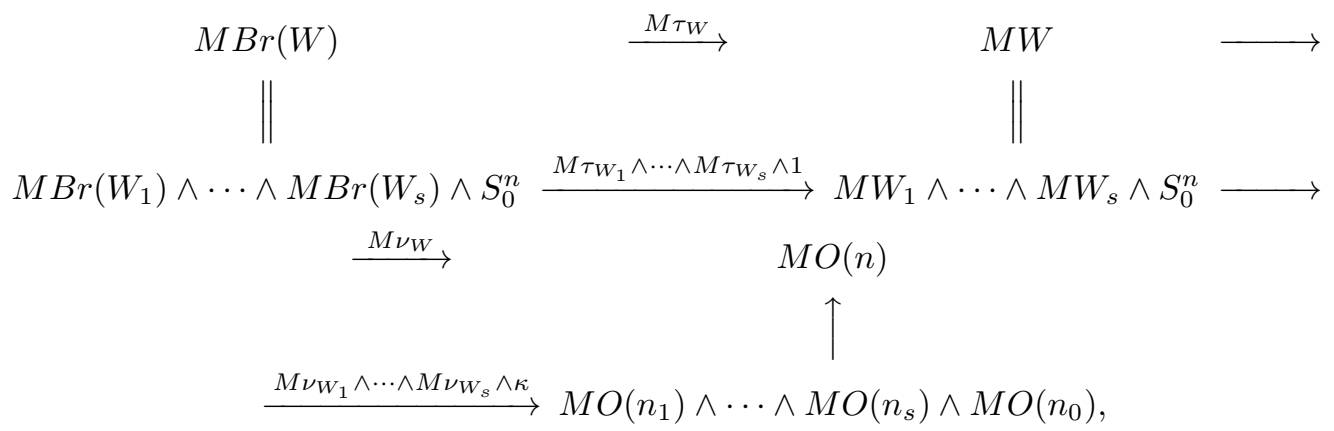

where $\kappa$ is the inclusion of the sphere $S^{n_{0}}$ into the Thom space: $S^{n_{0}} \rightarrow M O\left(n_{0}\right)$. We recall the definition of a Thom space (see [St], for example) and a spectrum $[\mathrm{Ad}],[\mathrm{Sw}]$. For a vector bundle $\xi: E \rightarrow B$ with a Riemannian metric we define the corresponding disc and spherical bundles $E_{D} \rightarrow B$ and $E_{S} \rightarrow B$. The Thom space $M(\xi)$ of the bundle $\xi$ is defined as the factor space $E_{D} / E_{S}$, which is the same as the one-point compactification of the space $E$. By definition, a spectrum $X$ is a sequence of cellular spaces $X_{n}, n \in \mathbb{Z}$ with base-point, provided with structure maps

$$
\epsilon_{n}: S X_{n} \rightarrow X_{n+1}, \quad n \in \mathbb{Z},
$$

where $S$ denotes the suspension of a space.

The composition of the maps $\tau_{W}$ and $\nu_{W}$ classifies the bundle

$$
Y_{W} \times_{W} R^{n} \rightarrow X_{W},
$$

where the space $Y_{W}$ was defined in Section 2. The Thom space of this bundle is equivalent to $Y_{W} \ltimes_{W} B^{n} / S^{n}$, where $B^{n}$ is the unit ball, and $\ltimes$ denotes the half smash product: $A \ltimes B=A \times B / A \times b_{0}\left(b_{0} \in B\right.$ is the base point). For the series $C$ it is equivalent to $Y_{C_{n}} \ltimes_{C_{n}} S^{1(n)}$, where $S^{1(n)}$ denotes the $n$-fold smash product of $S^{1}$, on which $C_{n}$ acts by permutations between copies of $S^{1}$ and by complex conjugation on each $S^{1}$. For the series $D$ the Thom space is equivalent to $Y_{C_{n}} \ltimes_{D_{n}} S^{1(n)}$, where $D_{n}$ also acts on $S^{1(n)}$ by permutations between copies of $S^{1}$ and by complex conjugation on each $S^{1}$, but according to the description of the group $D_{n}$ the number of conjugations must be even. If the Coxeter graph of a group consists of one point $\left(A_{1}=\Sigma_{2}=\mathbb{Z} / 2\right)$, then 
$B r_{2}=\mathbb{Z}$ and we have $B \Sigma_{2}=R P^{\infty}, B B r_{2}=S^{1}$ and $M \Sigma_{2}=S^{-1}\left(R P^{\infty}\right)\left(S^{-1}\right.$ denotes the inverse of the suspension functor $S$, which is invertable in the category of spectra), $M B r_{2}=S^{-1}\left(R P^{2}\right)$ and the map $M \tau$ is the canonical inclusion.

Using the procedure described above we get the Thom spectra $M W_{\infty}$ and $M B r\left(W_{\infty}\right)$ for a limit Coxeter group and the corresponding infinite braid group. This general situation of Thom spectra for Coxeter groups and generalized braid groups was considered in $[\mathrm{Ve}]$.

The pairings of Coxeter and braids groups generate the pairings of Thom spaces and spectra (which we shall denote by the same symbol $\mu$ ). It means in particular that the Thom spectra for the classical braid groups and generalized braid groups of type $C$ are multiplicative. There is a beautiful identification of the Thom spectrum for the classical braid group made by Mark Mahowald [Mah1, Mah2] and Fred Cohen [CF3].

THEOREM 6.1. The Thom spectrum of the braid group $M B r_{\infty}$ is multiplicatively isomorphic to the Eilenberg-MacLane spectrum $K(\mathbb{Z} / 2)$.

At first Mark Mahowald studied the following situation. Consider the map

$$
S^{1} \rightarrow B O
$$

realizing the generator of $\pi_{1}(B O) \cong \mathbb{Z} / 2$. Apply the functor $\Omega^{2} S^{2}$ to this map and consider the composition

$$
\eta: \Omega^{2} S^{3} \rightarrow \Omega^{2} S^{2}(B O) \rightarrow B O,
$$

where the second map is the retraction, arising from the infinite loop structure of $B O$. It was proved by Mark Mahowald [Mah1, Mah2] that the Thom spectrum of $\eta$ is equivalent to $K(\mathbb{Z} / 2)$. Then Fred Cohen [CF3] considered the composition

$$
B B r_{\infty} \rightarrow \Omega^{2} S^{3} \rightarrow B O,
$$

where the first map is that of G. Segal from the Theorem 4.11 and the second one is $\eta$. He proves that this composition is homotopic to $B \nu_{\infty}$ from (6.1) and that the Thom spectrum of this composition $M B r_{\infty}$ is multiplicatively isomorphic to the EilenbergMacLane spectrum $K(\mathbb{Z} / 2)$. The geometric aspects of the corresponding bordism theories were discussed by Fred Cohen [CF3] and Brian Sanderson [Sa].

The pairings described at the end of Section 2 induce on $M W_{\infty}$, for any limit Coxeter group $W_{\infty}$, at least one module structure over $M \Sigma_{\infty}$. The same way $M B r\left(W_{\infty}\right)$ has at least one module structure over $M B r_{\infty}$. Let $\kappa: S^{0} \rightarrow M B r_{\infty}$ be the unit map of the spectrum $M B r_{\infty}$. The composition of $1 \wedge \kappa$ and $\mu$ :

$$
\operatorname{MBr}\left(W_{\infty}\right) \wedge S^{0} \rightarrow \operatorname{MBr}\left(W_{\infty}\right) \wedge M B r_{\infty} \rightarrow \operatorname{MBr}\left(W_{\infty}\right)
$$

is equal to the identity map of $\operatorname{MBr}\left(W_{\infty}\right)$. This follows from the fact that the composition:

$$
W_{k}=W_{k} \times A_{0} \rightarrow W_{k} \times A_{l} \rightarrow W_{k+l+1}
$$

is equal to the inclusion $W_{k} \rightarrow W_{k+l+1}$. The same is true for $\operatorname{MBr}\left(W_{k}\right)$. Hence the spectrum $\operatorname{MBr}\left(W_{\infty}\right)$ is a direct summand in $\operatorname{MBr}\left(W_{\infty}\right) \wedge K(\mathbb{Z} / 2)$ and it is itself a wedge of Eilenberg-MacLane spectra. The spaces $X_{W}$ are connected, so $\pi_{0}\left(M B r\left(W_{\infty}\right)\right)=\mathbb{Z} / 2$. 
Analogously we prove that the spectrum $M \Sigma_{\infty}$ is equivalent to the wedge of EilenbergMacLane spectra $K(\mathbb{Z} / 2)$ being the module over $M B r_{\infty}$.

Shaun Bullet studied in $[\mathrm{Bu}]$ Thom spectra and corresponding bordism theories for the following groups: $\Sigma_{\infty}, \Sigma_{\infty} \imath \mathbb{Z} / 2=C_{\infty}, B r_{\infty} \imath \mathbb{Z} / 2$. It was proved by him that these bordism theories are multiplicative and that $M \Sigma_{*}, M(\Sigma \imath \mathbb{Z} / 2)_{*}$ and $M(B r \imath \mathbb{Z} / 2)_{*}$ are polynomial algebras over $\mathbb{Z} / 2$. He also proved that the canonical map induces the injective multiplicative morphism of cobordism theories:

$$
M \Sigma^{*}() \rightarrow M(\Sigma \imath \mathbb{Z} / 2)^{*}(),
$$

such that the composition

$$
M \Sigma^{*}() \rightarrow M(\Sigma \imath \mathbb{Z} / 2)^{*}(\mathrm{)}) \rightarrow M O^{*}(\mathrm{)},
$$

and the map

$$
M\left(\operatorname{Br}\{\mathbb{Z} / 2)^{*}() \rightarrow M O^{*}()\right.
$$

are surjective. Being a module over $M \Sigma_{\infty}$, the Thom spectrum $M W_{\infty}$, for a limit Coxeter group $W_{\infty}$, becomes a module over $K(\mathbb{Z} / 2)$ as well. So $M W_{\infty}$ is also a wedge of EilenbergMacLane spectra $K(\mathbb{Z} / 2)$. As a result we have the following theorem.

Theorem 6.2. The Thom spectra $M B r\left(W_{\infty}\right)$ and $M W_{\infty}$ for limit Coxeter groups are equivalent to the wedges of suspensions over the Eilenberg-MacLane spectrum $K(\mathbb{Z} / 2)$, $\pi_{0}\left(M B r\left(W_{\infty}\right)\right)=\mathbb{Z} / 2$

From the cofibre sequence

$$
S^{1} \rightarrow S^{1} \rightarrow R P^{2} \rightarrow \ldots,
$$

where the first map is multiplication by 2 , we obtain

COROLlaRY 6.1. If the Coxeter graph of $W_{\infty}$ contains an isolated vertex, $W_{\infty}=$ $W^{\prime} \times(\mathbb{Z} / 2)$, then $\operatorname{MBr}\left(W_{\infty}\right)=\operatorname{MBr}\left(W^{\prime}\right) \wedge S M B r\left(W^{\prime}\right)$, where $S$ denotes a suspension over a spectrum.

Now let us consider the Thom spectra for the groups $C_{\infty}$ and $D_{\infty}$. We would like to know the number of summands $K(\mathbb{Z} / 2)$ in each dimension for these spectra. This means to know modules $\pi_{*}\left(M B r\left(C_{\infty}\right)\right)=\operatorname{MBr}\left(C_{\infty}\right)_{*}$ and $\pi_{*}\left(M B r\left(D_{\infty}\right)\right)=\operatorname{MBr}\left(D_{\infty}\right)_{*}$. We use the knowledge of cohomology of the braid groups of the type $C$ and $D$ (Theorem 5.1) and then the Thom isomorphism.

Theorem 6.3. The Thom spectra $M B r\left(C_{\infty}\right)$ and $M B r\left(D_{\infty}\right)$ are equivalent to the following wedges of the Eilenberg-MacLane spectra

$$
\begin{gathered}
M B r\left(C_{\infty}\right)=\bigvee_{i=0}^{\infty} S^{i} K(\mathbb{Z} / 2) \\
M B r\left(D_{\infty}\right)=K(\mathbb{Z} / 2) \vee\left[\bigvee_{i=0}^{\infty} S^{2+i} K(\mathbb{Z} / 2)\right]
\end{gathered}
$$

The pairing defined for the braid groups of type $C$ induces a multiplicative structure (probably not commutative) for the theory $\operatorname{MBr}\left(C_{\infty}\right)_{*}()$. So $\operatorname{MBr}\left(C_{\infty}\right)_{*}$ has a 
ring structure which we would like to consider. We take the circle $S^{1}$ with its standard embedding in $\mathbb{R}^{n+1}$. Its normal bundle is trivial, so the corresponding classifying map

$$
\xi_{n}: S^{1} \rightarrow B O(n)
$$

is homotopic to zero. Now we take a fibration

$$
f_{n}: B C_{n} \rightarrow B O(n)
$$

homotopic to the canonical map, and analogously a fibration

$$
\psi: B B r\left(C_{n}\right) \rightarrow B C_{n}
$$

so that the composition

$$
f_{n} \psi=f_{n}^{\prime}: B B r\left(C_{n}\right) \rightarrow B O(n)
$$

is a fibration homotopic to the canonical map from $B B r\left(C_{n}\right)$ to $B O(n)$. We have

$$
\begin{gathered}
H_{1}\left(B B r\left(C_{n}\right) ; \mathbb{Z}\right)=B r\left(C_{n}\right) /\left[B r\left(C_{n}\right), B r\left(C_{n}\right)\right]=Z \oplus \mathbb{Z}, \\
H_{1}\left(B\left(C_{n}\right) ; \mathbb{Z}\right)=\operatorname{Br}\left(C_{n}\right) /\left[C_{n}, C_{n}\right]=\mathbb{Z} / 2 \oplus \mathbb{Z} / 2,
\end{gathered}
$$

and the map $H_{1}(\psi)$ is the canonical projection. We consider a map $g^{\prime}: S^{1} \rightarrow B B r\left(C_{n}\right)$, such that in homology the generator of $H_{1}\left(S^{1} ; \mathbb{Z}\right)$ maps by $H_{1}\left(g^{\prime}\right)$ to some generator $v$ of $H_{1}\left(B B r\left(C_{n}\right) ; \mathbb{Z}\right)$, and such that the composition

$$
f_{n}^{\prime} g^{\prime}: S^{1} \rightarrow B B r\left(C_{n}\right) \rightarrow B O(n)
$$

is homotopic to zero. We take $g: S^{1} \rightarrow B B r\left(C_{n}\right)$ as a map homotopic to $g^{\prime}$ and such that $f_{n}^{\prime} g=\xi_{n}$. The map $g$ defines a $\left(B B r\left(C_{n}\right), f_{n}^{\prime}\right)$-structure on $S^{1}$, and the map $\psi g$ defines a $\left(B C_{n}, f_{n}\right)$-structure on $S^{1}[\mathrm{St}]$. Let $w^{\prime} \in H^{1}\left(B B r\left(C_{n}\right) ; \mathbb{Z}\right)$ be the element dual to $v \in H_{1}\left(B B r\left(C_{n}\right) ; \mathbb{Z}\right)$ and $w$ is the reduction $\bmod 2$ of $w^{\prime}$. By our construction the characteristic number of $S^{1}$ with $\left(B B r\left(C_{n}\right), f_{n}^{\prime}\right)$-structure which corresponds to $w$, is a nonzero element of $\mathbb{Z} / 2$. So the bordism class of $S^{1}$ may be considered as a generator of $M B r\left(C_{\infty}\right)_{1}$ and its reduction from $B B r\left(C_{n}\right)$ to $B\left(C_{n}\right)$ is a nonzero element of $\left(M C_{\infty}\right)_{1}$. The ring $\left(M C_{\infty}\right)_{*}$ is a free commutative algebra over $\mathbb{Z} / 2$. So we have the following theorem.

THEOREM 6.4. The coefficient ring $M B r\left(C_{\infty}\right)_{*}$ of the bordism theory corresponding to the braid group of type $C$ is a polynomial algebra from one generator $s$ in dimension 1 :

$$
\operatorname{MBr}(C)_{*} \cong \mathbb{Z} / 2[s] .
$$

COROLlary 6.2. The image of the ring $\mathrm{MBr}\left(C_{\infty}\right)_{*}$ in the unoriented cobordism ring is equal to zero in positive dimensions.

R e m a rk. In the unoriented cobordism $\operatorname{ring} M O_{2}=\mathbb{Z} / 2, M O_{3}=0$. So the canonical map to unoriented cobordism for the bordism groups of the braids of type $D$

$$
\operatorname{MBr}\left(D_{\infty}\right)_{*} \rightarrow M O_{*},
$$

is neither a monomorphism nor an epimorphism.

Let us consider Thom spectra, corresponding to braid groups of finite Coxeter groups. We have seen that these spectra are smash products of spectra for irreducible Coxeter groups. Thom spectra $M B r_{k}$ were studied by E. Brown and F. Peterson [BP] and Ralph 
Cohen $[\mathrm{CR}$. Let $B(l)$ denote the Brown-Gitler spectrum [BG]. E. Brown and F. Peterson $[\mathrm{BP}]$ proved the following theorem.

TheOREM 6.5. The Thom spectrum $\mathrm{MBr}_{k}$ is 2-equivalent to the Brown-Gitler spectrum $B([k / 2])$, where $[a]$ denotes the integer part of a.

Corollary 6.3. If a morphism

$$
t_{n}: M B r_{n} \rightarrow K(\mathbb{Z} / 2)
$$

represents the generator of cohomologies of $\mathrm{MBr}_{n}$ as a module over the Steenrod algebra and $X$ is any $C W$ complex, then the corresponding morphism of generalized homology theories

$$
\left(M B r_{n}\right)_{q}(X) \rightarrow H_{q}(X, \mathbb{Z} / p)
$$

is surjective for $q \leq 2[n / 2]+1$.

For odd primes Ralph Cohen [CR] proved the following theorem.

TheOrem 6.6. For $p>2, M B r_{k p},(k \neq 10 \bmod p)$ is homotopy $p$-equivalent to the $(p-2) k$-fold suspension over the Brown-Gitler spectrum $S^{(p-2) k} B([k / p], p)$. If a morphism

$$
s_{k}: M B r_{k p} \rightarrow K(\mathbb{Z} / p,(p-2) k)
$$

represents the generator of cohomologies of $M B r_{k p}$ as a module over the Steenrod algebra, and $X$ is any $C W$ complex, then the corresponding morphism of generalized homology theories

$$
\operatorname{MBr}\left(C_{k p}\right)_{q+(p-2) k}(X) \rightarrow H_{q}(X, \mathbb{Z} / p)
$$

is surjective for

$$
q \leq \begin{cases}2 p([k / p]+1)-1, & \text { if } k \neq 0 \bmod p, \\ 2 k-1, & \text { if } k \equiv 0 \bmod p .\end{cases}
$$

Let $\Lambda_{p}$ be the mod p $\Lambda$-algebra described in [BCKQRS]. So $\Lambda_{2}$ is the graded $\mathbb{Z} / 2$ algebra generated by the elements $\lambda_{i}$ of degree $i$ for $i \geq 0$, which are subject to certain relations. If $p$ is odd, $\Lambda_{p}$ is the graded $\mathbb{Z} / p$-algebra generated by the elements $\lambda_{i-1}$ of degree $2 i(p-1)-1$ for $i \geq 1$, and the elements $\mu_{i-1}$ of degree $2 i(p-1)$ for $i \geq 0$, which are also subject to certain relations. Let $J_{k}$ be the left ideal of $\Lambda_{p}$ generated by $\lambda_{0}, \ldots, \lambda_{k-1}$ if $p=2$ and by $\lambda_{0}, \ldots, \lambda_{k-1}, \mu_{-1}, \ldots, \mu_{k-1}$ for $p$ odd. Then from the results of the papers [CR], [BCKQRS] we obtain the following facts.

COROLLARY 6.4. The 2-localization of the homotopy group $\pi_{q}\left(M B r_{n}\right)$ is isomorphic to $\left(\Lambda_{2} / J_{[n / 2]}\right)_{q}$ for $q<2[n / 2]$. The $p$-localization of the homotopy group $\pi_{q}\left(\operatorname{MBr}\left(C_{k p}\right)\right), k \not \equiv$ $0 \bmod p$, is isomorphic to $\left(\Lambda_{p} / J_{[k / p]}\right)_{q-(p-2) k}$ for $q<p(2[k / p]+k+2)-2(k+1)$.

We denote by $t_{W}$ the Thom class of the spectrum $M B r(W)$ :

$$
t_{W}: M B r(W) \rightarrow K(\mathbb{Z} / 2) .
$$

Let $\alpha_{C}: M B r_{n} \rightarrow M B r\left(C_{n}\right)$ be the map induced by the embeddings of Coxeter graphs which are described in Section 2. The composition:

$$
M B r_{n} \rightarrow M B r\left(C_{n}\right) \rightarrow M O(n) \rightarrow M O \rightarrow K(\mathbb{Z} / 2),
$$


where the last map is the Thom class of $M O$, is equal to the Thom class of $M B r_{n}$. The analogous compositions for the series $D$ and $E$ :

$$
\begin{gathered}
M B r_{n} \rightarrow M B r\left(D_{n}\right) \rightarrow M O(n) \rightarrow M O \rightarrow K(\mathbb{Z} / 2), \\
M B r_{n} \rightarrow M B r\left(E_{n}\right) \rightarrow M O(n) \rightarrow M O \rightarrow K(\mathbb{Z} / 2), n=6,7,8
\end{gathered}
$$

are equal to the Thom class of $M B r_{n}$. So we get that the homomorphisms induced in cohomology:

$$
\begin{gathered}
\left.H^{*}\left(M B r\left(C_{n}\right) ; \mathbb{Z} / 2\right) \rightarrow H^{*}\left(M B r_{n}\right) ; \mathbb{Z} / 2\right), \\
\left.H^{*}\left(M B r\left(D_{n}\right) ; \mathbb{Z} / 2\right) \rightarrow H^{*}\left(M B r_{n}\right) ; \mathbb{Z} / 2\right), \\
\left.H^{*}\left(M B r\left(E_{n}\right) ; \mathbb{Z} / 2\right) \rightarrow H^{*}\left(M B r_{n}\right) ; \mathbb{Z} / 2\right), n=6,7,8,
\end{gathered}
$$

are epimorphisms. Using the Corollary 6.3 we obtain the following theorem [Ve].

Theorem 6.7. If $X$ is any $C W$ complex then the maps for bordism theories $\operatorname{MBr}\left(C_{n}\right)_{*}(), \operatorname{MBr}\left(D_{n}\right)_{*}()$ and $\operatorname{MBr}\left(E_{n}\right)_{*}()$, induced by the Thom class $t$ :

$$
\begin{gathered}
\operatorname{MBr}\left(C_{n}\right)_{q}(X) \rightarrow H_{q}(X ; \mathbb{Z} / 2), \\
\operatorname{MBr}\left(D_{n}\right)_{q}(X) \rightarrow H_{q}(X ; \mathbb{Z} / 2), \\
\operatorname{MBr}\left(E_{n}\right)_{q}(X) \rightarrow H_{q}(X ; \mathbb{Z} / 2), n=6,7,8,
\end{gathered}
$$

are epimorphisms for $q \leq 2[n / 2]+1$.

Acknowledgements. The author is thankful to the Banach Center in Warsaw and the organizers of the Minisemester on Knot Theory, Professors Paweł Traczyk, Joanna Kania-Bartoszyńska and Józef H. Przytycki, for the invitation to take part in this very interesting and very well-organized minisemester.

\section{References}

[Ad] J. F. Adams, Stable homotopy and generalised homology, The University of Chicago Press, Chicago and London, 1974.

[Arn1] V. I. Arnold, The cohomology ring of colored braids, Mat. Zametki 5 No 2 (1969), 227-231 (Russian), English transl. in Trans. Moscow Math. Soc. 21 (1970), 30-52.

[Arn2] V. I. Arnold, On some topological invariants of algebraic functions, Trudy Moskov. Mat. Obshch. 21 (1970), 27-46 (in Russian), English transl. in Trans. Moscow Math. Soc. 21 (1970), 30-52.

[Art1] E. Artin, Theorie der Zopfe, Abh. math. semin. Univ. Hamburg 4 (1925), 47-72.

[Art2] E. Artin, Theory of braids, Ann. of Math. 48, No 1 (1947) 101-126.

[Bi] J. Birman, Braids, links, and mapping class groups, Ann. Math. Stud., No 82, 1974.

[Bo] N. Bourbaki, Groupes et algèbres de Lie. Chap. 4, 5, 6., Hermann, Paris, 1968.

[BCKQRS] A. Bousfield, E. Curtis, D. Kan, D. Quillen, D. Rector, J. Schlesinger, The mod $p$ lower central series and the Adams spectral sequence, Topology 5 (1966), $331-342$.

[Bri] E. Brieskorn, Sur les groupes de tresses, Sém. Bourbaki, n 401 , novembre 1971 (Lecture Notes in Math., No 317, 1973, 21-44). 
[BG] E. Brown, S. Gitler, A spectrum whose cohomology is a certain cyclic module over the Steenrod algebra, Topology 12 (1973), 283-295.

[BP] E. Brown, F. Peterson, The stable decomposition of $\Omega^{2} S^{r+2}$, Trans. Amer. Math. Soc. 243 (1978), 287-298.

[Bro] K. S. Brown, Cohomology of groups, Springer, N. Y. a. o., 1982.

[Bu] S. Bullett, Permutations and braids in cobordism theory, Proc. London Math. Soc. 38, Part 3 (1979) 517-531.

[Ch] W.-L. Chow, On the algebraical braid group, Ann. of Math. 49, No 3 (1948), 654-658.

[CF1] F. Cohen, Cohomology of braid spaces, Bull. Amer. Math. Soc. 79 No 4 (1973), 763-766.

[CF2] F. Cohen, Homology of $\Omega^{n+1} \Sigma^{n+1} X$ and $C_{n+1} X, n>0$, Bull. Amer. Math. Soc. 79 No 6 (1973), 1236-1241.

[CF3] F. Cohen, Braid orientations and bundles with flat connections, Invent. Math. 46 (1978), 99-110.

[CF4] F. Cohen, Artin's braid groups, classical homotopy theory, and other curiosities, Braids (Contemp. Math. 78, 1988), 167-206.

[CLM] F. Cohen, T. Lada, J. P. May, The homology of iterated loop spaces, (Lecture Notes in Math.; No 533), Springer-Verlag, Berlin a. o., 1976.

[CT] F. Cohen and L. Taylor, On the representation theory associated to the cohomology of configuration spaces, Algebraic Topology. Oaxtepec 1991, Contemp. Math. 146 (1993), 91-109.

[CR] R. Cohen, The geometry of $\Omega^{2} S^{3}$ and braid orientations, Invent. Math. 54 (1979), 53-67.

[D] P. Deligne, Les immeubles des groupes de tresses généralisés, Invent. Math. 17 (1972), 273-302.

[DL] E. Dyer and R. Lashof, Homology of iterated loop spaces, Amer. J. Math. 84 No 1 (1962), 35-88.

[FaN] E. Fadell and L. Neuwirth, Configuration spaces, Math. Scand. 10 Fasc. I (1962), 111-118.

[FoN] R. Fox and L. Neuwirth, The braid groups, Math. Scand. 10 Fasc. I (1962), 119-126.

[FK] R. Fricke, F. Klein, Vorlesungen über die Theorie der automorphen Functionen. Bd. I. Gruppentheoretischen Grundlagen, Teubner, Leipzig, 1897 (Johnson Repr. Corp., N. Y., 1965, 634 p.).

[F1] D. B. Fuks, Cohomology of the braid group mod 2, Funktsional. Anal. i Prilozh. 4, No 2 (1970), 62-75 (in Russian), English transl. in Functional Anal. Appl. 4 (1970), 143-151.

[F2] D. B. Fuks, Quillenization and bordisms, Funktsional. Anal. i Prilozh. 8, No 1 (1974), 36-42 (in Russian), English transl. in Functional Anal. Appl. 8 (1974), $31-36$.

[G1] V. V. Goryunov, Cohomology of the braid groups of the series C and $D$ and some stratifications, Funktsional Anal. i Prilozh. 12, No 2 (1978), 76-77 (in Russian), English transl. in Functional Anal. Appl. 12 (1978), 139-140.

[G2] V. V. Goryunov, Cohomology of the braid groups of the series $C$ and D, Trudy Moskov. Mat. Obshch. 42 (1981), 234-242 (in Russian), English transl. in Trans. Moscow Math. Soc. 1982, no 2. 
[H] A. Hurwitz, Über Riemannsche Flächen mit gegebenen Verzweigungspunkten, Math. Ann. 39 (1891), 1-61.

[La] S. Lambropoulou, Solid torus links and Hecke algebras of type B, in the Proceedings of the Conference on Quantum Topology, ed. D. N. Yetter, World Scientific Press, 1993, 225-245.

[Li] V. Ya. Lin, Artinian braids and groups and spaces connected with them, Itogi Nauki i Tekhniki (Algebra, Topologiya, Geometriya) 17 (1979), 159-227 (in Russian). English transl. in J. Soviet Math. 18 (1982) 736-788.

[Mah1] M. Mahowald, A new family in $\pi_{*}^{s}$, Topology 16 (1977), 249-254.

[Mah2] M. Mahowald, Ring spectra which are Thom complexes, Duke Math. J. 46, No 3 (1977), 249-259.

[May] J. P. May, The Geometry of iterated loop spaces, (Lecture Notes in Math.; No 271) Springer-Verlag, Berlin a. o., 1972.

[O] E. Ossa, On the cohomology of configuration spaces, Algebraic Topology: New Trends in Localization and Periodicity (Barcelona Conference on Algebraic Topology, 1994) Birkhäuser Verlag, Basel a. o., 1996, 353-361.

[Sa] B. Sanderson, The Geometry of Mahowald Orientations, in: Algebraic Topology. Aarhus, 1978 (Lecture Notes in Math., No 533) Springer-Verlag, Berlin a. o. (1979), 152-174.

[Se] G. Segal, Configuration spaces and iterated loop spaces, Invent. Math. 21 (1973), 213-221.

[St] R. E. Stong, Notes on cobordism theory, Princeton University Press, Princeton, 1968.

[Sw] R. M. Switzer, Algebraic Topology - Homotopy and Homology, Springer-Verlag Berlin a. o., 1975.

[Vai] F. V. Vainshtein, Cohomology of the braid groups, Funktsional. Anal. i Prilozh. 12, No 2 (1978), 72-73 (in Russian), English transl. in Functional Anal. Appl. 4 (1970), 143-151.

[Vas] V. A. Vassiliev, Complements of discriminants of smooth maps: topology and applications, (Translations of mathematical monographs; v. 98), AMS, Providence, 1992.

[Ve] V. V. Vershinin, Thom spectra of generalized braid groups, Preprint No 95/02-2, Université de Nantes, 1995. 